\title{
Unified Hydrodynamic Theory for Crystals, Liquid Crystals, and Normal Fluids
}

\section{Citation}

Martin, P. C., O. Parodi, and Peter S. Pershan. 1972. Unified hydrodynamic theory for crystals, liquid crystals, and normal fluids. Physical Review A 6(6): 2401-2420.

\section{Published Version}

doi:10.1103/PhysRevA.6.2401

\section{Permanent link}

http://nrs.harvard.edu/urn-3:HUL.InstRepos:10361959

\section{Terms of Use}

This article was downloaded from Harvard University's DASH repository, and is made available under the terms and conditions applicable to Other Posted Material, as set forth at http:// nrs.harvard.edu/urn-3:HUL.InstRepos:dash.current.terms-of-use\#LAA

\section{Share Your Story}

The Harvard community has made this article openly available.

Please share how this access benefits you. Submit a story.

Accessibility 


\title{
Unified Hydrodynamic Theory for Crystals, Liquid Crystals, and Normal Fluids*
}

\author{
P. C. Martin $\dagger$ \\ Lyman Labovatory of Physics, Harvard University, Cambridge, Massachusetts 02138 \\ and Laboratoire de Physique des Solides, Faculté des Sciences, 91-Orsay, France ${ }^{\dagger}$ \\ and Service de Physique Théorique, C.E.A. Saclay, Orme des Merisiers, \\ 91-Gif-sur-Yvette, France \\ and \\ O. Parodi \\ Laboratoire de Physique des Solides, Faculté des Sciences, 91-Orsay, France \\ and \\ P. S. Pershan $\S$ \\ Division of Engineering and Applied Physics, Harvard University, Cambridge, Massachusetts 02138 \\ and Laboratoire de Physique des Solides, Faculté des Sciences, 91-Orsay, France \\ (Received 31 May 1972)

\begin{abstract}
A unified hydrodynamic theory is presented that is appropriate for crystals; smectic,
\end{abstract} \\ cholesteric, and nematic liquid crystals; glasses; and normal fluids. In the theory, the in- \\ creased spatial degeneracy as the system progresses from crystalline and mesomorphic \\ phases to the isotropic fluid phase is marked by successive reductions in the number of first- \\ order elastic constants and in the number of transport coefficients. Distinction between local \\ lattice dilations and local mass changes, and recognition of processes like vacancy diffusion \\ that this difference makes possible, are crucial for understanding the connection between \\ theories in different phases. Formulas are derived that give the number of hydrodynamic \\ modes and the frequencies, lifetimes, and intensities of these modes in all of the above sys- \\ tems. In the nematic and cholesteric phases, the results agree with some found previously. \\ In more complex systems, they are new. An attempt is made to explain the differences be- \\ tween the present hydrodynamic theory and other phenomenological proposals.
}

\section{INTRODUCTION}

When a system with many degrees of freedom is disturbed from a thermal-equilibrium state and then allowed to relax, almost every degree of freedom will relax to its equilibrium value in a time determined by and characteristic of the system's detailed microscopic interactions. There will, however, be a few collective modes which will decay more slowly. More specifically a few will decay in times proportional to some power of their wavelength. Hydrodynamics describes these "long-lived" degrees of freedom or modes. The existence of such modes can always be traced either to conservation laws or, in the case of ordered systems, to "continuous broken symmetries," since many-body systems are too chaotic for slow relaxation to occur "accidentally."

Let us illustrate by example: Sound waves and thermal conduction in a simple fluid ${ }^{1}$ have characteristic frequencies ( inverse times) which vanish as the wavelength goes to infinity because of the conservation laws for energy, momentum, and density. On the other hand, the long-wavelength spin waves in an isotropic antiferromagnet have hydrodynamic behavior ${ }^{2}$ by virtue of the continuous rotational symmetry of the spin system (relative to the lattice). That is, the system has equal en- ergy for all orientations of the staggered spin arrangement relative to the lattice.

In contrast to the orientation of the staggered magnetization which is a hydrodynamic variable in the isotropic antiferromagnet, the magnitude of the staggered magnetization is not. If disturbed from equilibrium the magnitude will decay, in microscopic times, to a value characteristic of a local thermal equilibrium, following which further decay will be completely described by the hydrodynamic decay of temperature, density, etc. Thus although the magnitude of the magnetization decays slowly, it is not a new independent hydrodynamic variable. Changes in the magnitude of the magnetization do not change the symmetry of the equilibrium state, and since they couple to the total energy and density in microscopic times they do not lead to new independent long-lived degrees of freedom.

Since the hydrodynamic properties of a system follow from the conservation laws and broken symmetries (for ordered systems) they can be derived from the thermodynamic properties of the system under the single assumption that the slowly varying time derivatives at small wave number have first, and in some cases second, derivatives with respect to wave number (i.e., that spatial gradients exist). This assumption is by no means com- 
pletely trivial; there are indications that hydrodynamics, in the sense used here, does not exist for certain systems. For example, it has been argued that the heat current and stress tensor are not proportional to gradients of temperature and velocity in two dimensions. Consequently, viscosity and thermal conductivity do not exist. Also, in two dimensions there are systems which seem to "order" in the sense that they undergo a phase transition, but in which we know for certain that the order parameter vanishes ${ }^{3}$ (e.g., a two-dimensional Heisenberg magnetic system).

This problem is not an academic one. There are strong argument $s^{4,5}$ that indicate that precisely the same phenomenon occurs in three-dimensional cholesteric and smectic liquid crystals. The difficulties produced by such a failure are hard to assess. The likelihood that a gradient expansion is rigorously valid is certainly reduced. Nonetheless, we would expect, and experiments on twodimensional Heisenberg systems suggest, that the failure may not be serious, especially for wavelengths small compared to the size of a container that stabilizes the order.

The point of view summarized here has been previously utilized in normal fluids, ${ }^{1}$ mixtures of normal fluids, ${ }^{6}$ superfluids, ${ }^{7}$ ferromagnets and antiferromagnets, ${ }^{2,8}$ and nematic ${ }^{8}$ and cholesteric liquid crystals. ${ }^{5}$ Although such discussions give no estimate for the magnitude of the parameters that are introduced, they do predict the long-wavelength long-time behavior of systems with as much rigor as more detailed presently feasible microscopic approaches. They are only rigorously applicable to those ordered fluids which have a hydrodynamic regime. For those that do not, we believe that a rigorous description is impossible but that it is not unreasonable to hope that our equations will be quantitatively correct for all but the longest wavelengths.

Subject only to this qualification, we present here a unified picture of the hydrodynamics of systems that exhibit spatial order. From it we shall retrieve the results that have been obtained previously for nematic and cholesteric liquid crystals and also derive for the first time the corresponding properties of smectic liquid crystals and ordinary crystals. An important conclusion of our work is that all ordered systems contain extra dissipative processes (and thus extra transport parameters) in addition to the thermal conductivities and viscosities of simple fluids. In crystals ${ }^{10}$ these processes are the diffusion and thermal diffusion of point defects or vacancies. It would be interesting to search for their effects in both crystals and smectic liquid crystals. It is also quite possible that some observed low-temperature properties of glasses and radiation-damaged crystals might be explained in these terms.

The present paper demonstrates that the properties of all fluids (simple liquids as well as liquid crystals) can be obtained by relaxing (i.e., setting equal to zero) properties which are present in a complicated crystal. This was the prejudice that prompted an earlier incomplete attempt ${ }^{11}$ to explain liquid crystals. It appears that the prejudice was correct and the effort failed fundamentally because the crystal was not treated generally enough.

In the present paper we shall eschew certain mathematical machinery involving correlation functions, ${ }^{1,12}$ which would make a number of derivations clearer and lead to a number of additional results. The reader interested in calculating the intensities of modes is therefore left with some cumbersome exercises. In compensation, the formulas which we shall exhibit will be relatively brief and hopefully comprehensible.

\section{GENERAL CONSIDERATIONS}

As mentioned above the first task in developing any hydrodynamic theory is to list the independent hydrodynamic variables. Corresponding to conservation of mass, momentum, and energy, there are the mass density $\rho$, the momentum density $\overrightarrow{\mathrm{g}}$, and the energy density $\epsilon$, which satisfy the conservation equations

$$
\dot{\rho}=-\nabla_{i} g_{i}, \quad \dot{g}_{i}=-\nabla_{j} \sigma_{i j}, \quad \dot{\epsilon}=-\nabla_{i} J_{i}{ }^{\epsilon},
$$

where $\sigma_{i j}$ is the stress tensor and $J_{i}{ }^{\epsilon}$ is the energy current density. We take $\sigma_{i j}=\sigma_{i j}$ and reserve further discussion on this point for Appendix A.

All quantities are the averages of the microscopic expressions for the state in question in the fixed "laboratory" space-time frame. ${ }^{13}$ The assumption that a gradient expansion is valid is invoked by asserting that for any slowly varying fluctuation, with wave vector $\vec{q}$, the currents on the right-hand side of Eqs. (2.1) all go to zero as some power of $q$ in the local rest system of the fluid. Thus, in the long-wavelength limit the characteristic frequencies (e.g., $\dot{\rho}=-i \omega \rho$ ) all vanish. For an ordinary fluid these are all the equations and one proceeds by expanding the currents in Eqs. (2.1) about thermal equilibrium, so as to obtain five (because there is a separate equation for each component of $\overrightarrow{\mathrm{g}}$ ) simultaneous differential equations, first order in $d / d t$. Substituting $-i \omega=d / d t$ in these equations leads to a deter minantal equation

$$
\sum_{n=0}^{5} A_{n}(\overrightarrow{\mathrm{q}}) \omega^{n}=0
$$

in which the polynomials in $\overrightarrow{\mathrm{q}}, A_{n}(\overrightarrow{\mathrm{q}})$ depend on the wave vector. To lowest order in $q$ two of the five roots of this equation are of the form $\omega= \pm c q$ and describe propagating sound. The other three have 
the form $\omega=i D q^{2}$ and describe the diffusive behavior of thermal relaxation and of the two nonpropagating transverse shear waves of an ordinary fluid. It is worth noting here a general property of any hydrodynamic theory: Propagating modes must occur in pairs. This requirement is a consequence of time-reversal invariance.

The five equations (2.1) that apply in any system are not sufficient when the system has some symmetry that can be continuously broken. For example, in the isotropic antiferromagnet discussed above ${ }^{2}$ the orientation of the staggered magnetization (and similarly, in a nematic liquid crystal, the director) can point in any direction. Consequently a slow (continuous) spatial variation in the direction will require little energy and since the energy required to produce a deviation without allowing the system to readjust is greater than the energy associated with the mode, the time rate of change of the variable that describes the "broken symmetry" must be small and the variable hydrodynamic. For the nematic liquid crystal $^{9}$ this argument implies that there are two additional variables and so there are two equations beside Eqs. (2.1). There are therefore seven hydrodynamic modes for a nematic liquid crystal. Note also that the extra variables are even under timereversal symmetry. This is not always the case. In particular, in a superfluid the extra hydrodynamic variable is the superfluid ${ }^{7}$ velocity which is odd under time reversal. The continuous broken symmetry for superfluidity is related to gauge invariance. Since the three components of the superfluid velocity are given in terms of the gradients of a single variable, the superfluid phase, there is just one extra equation to add to Eqs. (2.1). In place of the fifth-order equation for the characteristic frequencies of a normal fluid, the superfluid has a sixth-order equation and the possibilities are all propagating modes $\omega= \pm c_{1} q, \pm c_{2} q$, $\pm c_{3} q$; two pairs of propagating modes $\omega= \pm c_{1} q$, $\pm c_{2} q$ and two of the diffusive type $\omega=i D_{1} q^{2}, i D_{2} q^{2}$; etc. Further analysis is necessary to determine which possibility occurs, e.g., to conclude that in helium the second case, corresponding to first and second sounds, is realized.

Before going further we would like to emphasize what we have illustrated above - that the most simple and fundamental quantity in any hydrodynamic theory is the number of independent degrees of freedom the theory contains. As we have seen above this number is an invariant property of the system and it determines directly the number of modes one will find in that system. Furthermore, since propagating modes always occur in pairs, having counted the modes, we can readily list the possible types of solutions for any system. Starting from the simple liquid with two propagating solutions and three diffusive, the addition of one new variable will either eliminate one of the previous diffusive modes in favor of a pair of propagating modes, as in the superfluid, ${ }^{7}$ or it will add a further diffusive mode as in a normal binary fluid mixture. We shall see below that for most directions of $\vec{q}$ the mode structure of smectic $A$ and cholesteric liquid crystals resembles the mode structure in a superfluid (i. e., two pairs of propagating and two diffusive modes) but that in special symmetry directions, there are, as in a binary fluid mixture, four diffusive and one pair of propagating modes. ${ }^{14}$ The same will be demonstrated for smectic $C$ phases except that the latter have an additional diffusive mode. Finally, we shall argue that crystalline solids have three extra hydrodynamic variables so that there are, in general, three pairs of propagating sound waves (six solutions), one diffusive mode for thermal conductivity, and (for a monomolecular crystal) one extra, corresponding to diffusion of vacancies. ${ }^{15}$ In all, there are eight solutions. Except in special directions the mode structure of a smectic $B$ phase is the same as that of a crystalline "solid." 16

Having counted the number of hydrodynamic variables, and consequently the number of modes, it remains to develop mathematical expressions that will describe the physically observable properties of the modes. Since we are considering a theory that is applicable only in the limiting case of $\omega$ $\rightarrow 0$ we can exploit the fact that irreversible or dissipative effects can be made arbitrarily small by taking $q$ arbitrarily small. Strictly speaking, in the absence of externally applied forces and in the limit of vanishingly slow temporal changes all hydrodynamic motions are thermodynamically reversible and adiabatic (i.e., entropy is conserved). Dissipative or irreversible effects only enter a hydrodynamic theory at the next order in frequency and wave vector. This property is of course intimately related to the connection between thermodynamics and hydrodynamics to which we have alluded. The hydrodynamic regime is the regime in which every process takes place slowly compared to any microscopic time. For such slow processes the dissipation in modes with $\omega \tau \ll 1$ will be arbitrarily small. Only those terms for which $\tau$ is inversely proportional to $q$ contribute, and their contribution vanishes with $q$. If we were to try to include some of the processes which occur in microscopic times and to stop short of a detailed microscopic calculation we would have to present some microscopic argument for including only those processes we chose to single out (i.e., some reason for expecting their decay to be exponential and their lifetime longer than others we chose to ignore). It is confusion on this point which has led to the inappropriate "generalizations"17 
of the hydrodynamic equations of nematic liquid crystals.

Having argued that for specific systems there can be variables in addition to the conserved ones which are both independent and "slow" we proceed by adding to Eqs. (2.1) equations like

$$
\dot{x}^{\alpha}=J^{\alpha},
$$

where $\left\{x^{\alpha}\right\}$ are the extra independent hydrodynamic variables and the $\left\{J^{\alpha}\right\}$ describe their decay. Asserting that there is an equilibrium state with an order parameter is equivalent to saying there is a thermodynamic identity of the form

$$
T d(\rho s)=d \epsilon-\mu d \rho-v_{i} d g_{i}-\sum_{\alpha} F^{\alpha} d x^{\alpha},
$$

where $\epsilon$ is the energy density of the system in the laboratory frame. If $\epsilon^{0}$ is the energy density of the system in its rest frame (moving with velocity $v_{i}$ relative to the laboratory frame), then

$$
\begin{aligned}
& g_{i}=\rho v_{i}, \quad \epsilon=\epsilon^{0}+\frac{1}{2} \rho v^{2}, \\
& \rho \mu=p+\epsilon^{0}-T \rho s-\frac{1}{2} \rho v^{2} .
\end{aligned}
$$

The quantities $p$ and $s$ are the thermodynamic pressure and the entropy in the rest frame of the system and $\mu$ is the chemical potential in the laboratory frame. In thermal equilibrium, in the absence of externally applied forces, $F^{\alpha}=0$. Equation (2.3) represents a prescription for calculating the work done on the system when slow hydrodynamic external forces $\delta T, \delta \mu$ (or $\delta p$ ), $\delta \overrightarrow{\mathrm{v}}$, and $\delta F^{\alpha}$ are applied to it. The "forces" $F^{\alpha}$ that appear in (2.3) must approach zero as the wave vector $\vec{q}$ tends to zero since this was just the criterion by which we admitted $\left\{x^{\alpha}\right\}$ as hydrodynamic variables. A convenient way to incorporate this requirement is to write

$$
F^{\alpha}=-\nabla_{i} \phi_{i}^{\alpha}
$$

and through a redefinition of $\epsilon$,

$$
T d(\rho s)=d \epsilon-\mu d \rho-v_{i} d g_{i}-\sum_{\alpha} \phi_{i}^{\alpha} d\left(\nabla_{i} x^{\alpha}\right) .
$$

[In a superfluid, $x$ is the superfluid velocity potential (or phase), $\vec{\nabla} x$ is the (irrotational) superfluid velocity $\overrightarrow{\mathrm{v}}_{s}$, and $\phi_{i}$ is $\rho_{s} v_{s i}$ in the frame in which the whole fluid is at rest.] For thermal equilibrium in the absence of external forces,

$$
\rho d\left(\mu+\frac{1}{2} v^{2}\right)=d p-\rho s d T,
$$

and for hydrodynamic fluctuations about thermal equilibrium we define the nonequilibrium quantities $T(\overrightarrow{\mathrm{r}}, t), \mu(\overrightarrow{\mathrm{r}}, t)$ or $p(\overrightarrow{\mathrm{r}}, t), v_{i}(\overrightarrow{\mathrm{r}}, t)$, and $\left\{\phi_{i}{ }^{\alpha}(\vec{r}, t)\right\}$ to be the same functions of the local mass, energy, and momentum densities and order parameter as they are in equilibrium,

$$
\rho \nabla_{i}\left(\mu+\frac{1}{2} v^{2}\right)=\nabla_{i} p-\rho s \nabla_{i} T .
$$

Restricting attention to the arbitrary slow hy- drodynamic changes in $\epsilon, \rho, g$, and $\left\{x^{\alpha}\right\}$, we can rewrite Eq. (2.6) as

$$
T \frac{\partial}{\partial t}(\rho s)=\dot{\epsilon}-\mu \dot{\rho}-v_{i} \dot{g}_{i}-\sum_{\alpha} \phi_{i}^{\alpha} \nabla_{i} \dot{x}^{\alpha},
$$

where all time derivatives are calculated in the laboratory frame. The observation that the work that would be done on the system by external forces $\delta \mu, \delta v, \delta \phi, \delta T$ must be positive if the system is stable and the recognition that in this case the work would be done at the rate

$$
T \frac{d}{d t} \int d \overrightarrow{\mathbf{r}}[\rho s] \equiv R
$$

imply that $R \geq 0 .{ }^{18}$

In the absence of dissipative effects, that is, in the limit as $\vec{q} \rightarrow 0$, the hydrodynamic motions are reversible adiabatic motions; $R$ differs from zero only to the extent that $\vec{q} \neq 0$ when dissipation is taken into account. Thus Eq. (2.10) actually constitutes a definition of the entropy changes accompanying irreversible nonequilibrium motions. These changes can be expressed in terms of thermodynamic variables because a "gradient expansion" is possible for hydrodynamic motions. Substituting Eqs. (2.1) and (2.2) and using definitions (2.4), (2.8), and (2.10), we obtain

$$
\begin{aligned}
& R=-\int d \overrightarrow{\mathrm{r}}\left\{(1 / T)\left[J^{\epsilon}-\left(p+\epsilon^{0}\right) v\right]_{i} \nabla_{i} T\right. \\
&\left.-v_{i}\left(\nabla_{j} \sigma_{i j}-\nabla_{i} p\right)+\sum_{\alpha} \phi_{i}^{\alpha} \nabla_{i} J^{\alpha}\right\} \\
&+ \text { (terms of order } v^{3} \text { and terms of higher } \\
& \geq 0 . \\
& \text { order in the gradient) }
\end{aligned}
$$

Henceforth we shall consider only small deviations from a state in equilibrium and at rest. We shall therefore drop all terms not given explicitly in Eq. (2.11) and retain only the terms that are linear in the small fluctuating quantity $v_{i}$.

For a normal fluid, in which there is no order parameter, there are no $\phi_{i}{ }^{\alpha}$ terms and the reversible parts of $J_{i}^{\epsilon}=\left(p+\epsilon^{0}\right) v_{i}$ can be obtained by arguments on Galilean invariance. The reversible part of the stress tensor satisfies $\nabla_{j} \sigma_{i j}=\nabla_{i} p$ so that for adiabatic reversible motion $\sigma_{i j}=\delta_{i j} p$. Irre versible effects are taken into account by writing

$$
\begin{aligned}
& \left(J_{i}{ }^{\epsilon}\right)^{D} \equiv J_{i}{ }^{\epsilon}-\left(p+\epsilon^{0}\right) v_{i}, \\
& \nabla_{j} \sigma_{i j}{ }^{D} \equiv \nabla_{j} \sigma_{i j}-\nabla_{i} p,
\end{aligned}
$$

and, including only terms quadratic in the deviations from equilibrium,

$$
R \approx-\int d \overrightarrow{\mathrm{r}}\left[T^{-1}\left(\nabla_{i} T\right)\left(J_{i}^{\epsilon}\right)^{D}-\left(\nabla_{i} v_{j}\right) \sigma_{j i}^{D}\right] .
$$

The positive definiteness of $R$, corresponding to the fact that energy is dissipated, is guaranteed by writing for the normal fluid

$$
\left(J_{i}^{\epsilon}\right)^{D}=-\kappa_{i j} \nabla_{j} T
$$




$$
\left(\sigma_{i j}\right)^{D}=-\eta_{i j k l} \nabla_{l} v_{k} .
$$

$\kappa_{i j}$ and $\eta_{i j k l}$ are the usual thermal conductivity and viscosity of a normal fluid. Note that there are no cross terms $\left(J_{i}{ }^{6}\right)^{D} \sim \nabla_{k} v_{l}$, etc. This is a special case of a fundamental property of the equations of motion. Irreversible effects appear in the equations of motion with opposite time-reversal properties to reversible effects. Since $\epsilon$ is even under time reversal the reactive parts must be proportional to odd variables $\left[\left(J_{i}{ }^{\epsilon}\right)^{R} \equiv\left(p+\epsilon^{0}\right) v_{i}\right]$ and the dissipative parts to even ones $\left[\left(J_{i}{ }^{6}\right)^{D}\right.$ $\left.=-\kappa_{i j} \nabla_{j} T\right]$. There is no possibility for cross terms. Note also that because the mass current is conserved, there are no dissipative coefficients linking $\overrightarrow{\mathrm{J}}^{\epsilon}$ with $\vec{\nabla} p$ and $\overrightarrow{\mathrm{g}}$ with $\vec{\nabla} T$. Note finally that reactive terms coupling $\vec{J}^{\epsilon}$ with $\nabla \overrightarrow{\mathrm{V}}$ and $\vec{\sigma}$ with $\vec{\nabla} T$ are of higher order in the gradient than we need consider and that, furthermore, in a simple fluid they are ruled out by considerations of spatial symmetry.

As we noted, $R$ represents the irreversible work that would have to be done on the system by external "forces" if the hydrodynamic fluctuations were sustained, rather than allowed to decay. For an ordered system, again to second order in fluctuations from equilibrium, we have

$$
\begin{aligned}
R=\int d \overrightarrow{\mathrm{r}}\left(-T^{-1}\left(\nabla_{i} T\right)\left(J_{i}{ }^{\epsilon}\right)^{D}-\right. & \left(\nabla_{i} v_{j}\right)\left(\sigma_{j i}\right)^{D} \\
& \left.+\sum_{\alpha}\left(\nabla_{i} \phi_{i}{ }^{\alpha}\right)\left(J^{\alpha}\right)^{D}\right) .
\end{aligned}
$$

Taking into account the time-reversal property of dissipative effects and recognizing that from a phenomenological point of view all definitions of $\left(\vec{J}^{\epsilon}\right)^{D},(\vec{\sigma})^{D}$, and $\left(J^{\alpha}\right)^{D}$ that lead to the same $R$ are physically equivalent, we can immediately write

$$
\begin{aligned}
& \left(\sigma_{i j}\right)^{D}=-\eta_{i j k l} \nabla_{i} v_{k}, \\
& \left(J_{i}\right)^{D}=-\kappa_{i j} \nabla_{j} T-\sum_{\alpha} \xi_{i}{ }^{\alpha}\left(\nabla_{j} \phi_{j}{ }^{\alpha}\right), \\
& \left(J^{\alpha}\right)^{D}=T^{-1} \xi_{i}{ }^{\alpha} \nabla_{i} T+\sum_{\alpha} \Gamma^{\alpha \alpha^{\prime}}\left(\nabla_{i} \phi_{i}{ }^{\alpha}\right),
\end{aligned}
$$

where $\Gamma^{\alpha \alpha^{\prime}}=\Gamma^{\alpha^{\prime} \alpha}$.

We shall show explicitly in Appendix A that with no loss in generality we can always take $\sigma_{i j}=\sigma_{j i}$ and thereby conveniently include all consequences of angular momentum conservation. Accepting this fact, we see that we can describe the dissipation by two symmetric positive definite matrices. The first is a $6 \times 6$ matrix for the viscosities whose rows and columns are labeled by the indices of $\sigma_{i j}(11,22,33,12,23,31)$. The second is a $(3+n) \times(3+n)$ positive definite matrix whose rows and columns are labeled by the three components of the heat current and the $n$ components $\left\{\partial x^{\alpha} / \partial t\right\}$. When there is a single order parameter, as is the case in a smectic $A$ phase, this matrix is $4 \times 4$ $(n=1)$. For a smectic $C$ phase, on the other hand, $n=2$ and the dissipative matrix is $5 \times 5$. For a crystal, $n=3$. In a superfluid the phase variable is odd under time reversal; as a result there are no additional dissipative cross terms of the type discussed above but instead additional viscosities.

Having obtained the dissipative parts of $\vec{J}^{\epsilon}, \vec{\sigma}$, and $\left\{J^{\alpha}\right\}$ it only remains to specify how the reversible parts of these quantities depend on $\delta \rho, \delta \epsilon, \delta \overrightarrow{\mathrm{g}}$, and $\left\{\delta x^{\alpha}\right\}$, or their conjugate variables $\delta \mu$ or $\delta p$, $\delta T, \overrightarrow{\mathrm{v}}$, and $\phi^{\alpha}$.

The reversible parts of the momentum density $\overrightarrow{\mathrm{g}}$ and energy current $\vec{J}^{\epsilon}$ can be deduced by using Galilean invariance arguments [Eqs. (2.4) and (2.12)]. To the required order in $v$ and gradients they are unchanged by the introduction of the variables $\left\{x^{\alpha}\right\}$. Since the $\left\{x^{\alpha}\right\}$ considered here are all even under time reversal the most general form for the $\left\{J^{\alpha}\right\}$ is

$$
J^{\alpha}=A_{i}^{\alpha} v_{i}+A_{i j}{ }^{\alpha} \nabla_{j} v_{i}+\cdots .
$$

For the problems we shall be discussing here the $\left\{x^{\alpha}\right\}$ will always be some components of vectors. In a smectic $A$ phase, for example, the "broken symmetry" is translational, ${ }^{14}$ that is, a small translation along the normal to the planes produces a different, but equivalent, state of the system. If we take the 3 axis in this direction then the only nonvanishing component of the single order parameter $A_{i}$ is $A_{3}$, and $\dot{x}=A_{3} v_{3}$. Since nothing has been said about the dimensions or magnitude of the $\left\{x^{\alpha}\right\}$ we can, with no loss in generality, take $A_{3}=1$ and write $J=v_{3}$.

In a nematic phase, the broken symmetry is rotational, not translational. With this type of order a uniform displacement of such a system does not change the state, so that $\left\{A_{i}{ }^{\alpha}\right\}=0$, and $J^{\alpha}$ $=A_{i j}{ }^{\alpha} \nabla_{j} v_{i}$. The conventional representation for a nematic phase is one in which $\left\{x^{\alpha}\right\}$ is identified with $\delta n_{\alpha}(\alpha=1,2)$ and

$$
\delta \dot{n}_{i}=\mu_{i j k} \partial_{k} v_{j}=\frac{1}{2} \lambda\left(\partial_{i} v_{3}+\partial_{3} v_{i}\right)-\frac{1}{2}\left(\partial_{i} v_{3}-\partial_{3} v_{i}\right) .
$$

In this case $A_{i}{ }^{\alpha}=0$ and the quantity $A_{j k}{ }^{i}$ called $\mu_{i j k}$ is chosen so that $\mu_{i j k}=\frac{1}{2}(\lambda-1) \delta_{i k} \delta_{j 3}$ $+\frac{1}{2}(\lambda+1) \delta_{i j} \delta_{k 3}$. The quantity $\lambda$ is conventionally written as $\lambda \equiv-\gamma_{2} / \gamma_{1}$ in the literature ${ }^{19,20}$ of nematic phases with $\gamma_{1}{ }^{-1}$ the dissipation coefficient $\Gamma$ of (2.15). Discussion in Appendix B is directed towards differentiating between the physical significance of $\gamma_{1}$ and $\gamma_{2}$.

The effects of the $\left\{x^{\alpha}\right\}$ on the reactive part of $\vec{\sigma}$, that is, on the reactive part of $\vec{g}$ follow immediately from the fact that they can contribute nothing to $R$ [Eq. (2.11) or (2.9)]. Thus, if Eq. (2.16) leads to reactive terms $\sum_{\alpha} \phi_{k}{ }^{\alpha} \nabla_{k} A_{i j}{ }^{\alpha} \nabla_{j} v_{i}$ in (2.11), others in $\dot{g}_{i} \sim \sum_{\alpha} A_{i j}{ }^{\alpha} \nabla_{j}\left(\nabla_{k} \phi_{k}{ }^{\alpha}\right)$ must cancel them. The cancellation must occur since the reactive terms, resulting from expansion about thermal equilibrium, can be obtained from thermodynamic derivatives, i.e., 


$$
\left(\frac{\partial \dot{x}^{\alpha}}{\partial v_{k}}\right)_{T, p, \phi}=\left(\frac{\partial \dot{g}_{k}}{\partial\left[\nabla_{i} \phi_{i}^{\alpha}\right]}\right)_{T, p, \vec{v}} .
$$

To be more specific on this point, the hydrodynamic fluctuations, satisfying Eq. (2.6) and definitions (2.4) and (2.7), also satisfy

$d(\epsilon / \rho)=-p d(1 / \rho)+\rho^{-1} g_{i} d v_{i}+\rho^{-1} \sum_{\alpha} \phi_{i}{ }^{\alpha} \nabla_{i} d x^{\alpha}+T d s$.

(2.19a)

Alternatively, neglecting a divergence term that would contribute only to surface energies, to second order in deviations from thermal equilibrium, we have

$$
\begin{aligned}
& d\left(\epsilon^{\prime} / \rho\right)=-p d(1 / \rho)+\rho^{-1} g_{i} d v_{i} \\
& +\rho^{-1} \sum_{\alpha} x^{\alpha} d \nabla_{i} \phi_{i}{ }^{\alpha}+T d s, \\
& \epsilon^{\prime} \equiv \epsilon+\sum_{\alpha} x^{\alpha} \nabla_{i} \phi_{i}{ }^{\alpha} .
\end{aligned}
$$

The reactive, or nondissipative parts, of the hy drodynamic variations can always be discussed by treating the limit in which $\vec{q}$ approaches zero. Thus, from Eq. (2.19b), all the reactive parts of the hydrodynamic variations satisfy the crossderivative relations

$$
\frac{\partial g_{i}}{\partial\left(\nabla_{j} \phi_{j}^{\alpha}\right)}=\frac{\partial x^{\alpha}}{\partial v_{i}} \text {. }
$$

To the necessary order, the independent variables held constant in Eq. (2.20) can be chosen in several different fashions. Equation (2.18) follows from Eq. (2.20) for hydrodynamic variations.

Thus with the definitions of the nonequilibrium quantities $p,\left(\nabla_{i} \phi_{i}{ }^{\alpha}\right)$, and $T$ [Eqs. (2.7) and (2.19a)] in terms of the microscopically defined variables $\rho, x^{\alpha}, \in$ (or, with a suitable definition, $s$ in place of $\epsilon$ ), all of the reactive parts of $\dot{\mathrm{g}}, \dot{\epsilon}$, and $\dot{x}^{\alpha}$ can be specified in terms of the $\left\{A_{i j}{ }^{\alpha}\right\}$ and the symmetric matrix of thermodynamic derivatives

$$
\left[\begin{array}{ccc}
\rho^{2}\left(\frac{\partial p}{\partial \rho}\right)_{s, x^{\alpha}} & \rho^{2}\left(\frac{\partial T}{\partial \rho}\right)_{s, x^{\alpha}} & \rho\left(\frac{\partial \phi_{j}^{\alpha}}{\partial \rho}\right)_{s, x^{\alpha}} \\
\left(\frac{\partial p}{\partial s}\right)_{x^{\alpha}, \rho} & \left(\frac{\partial T}{\partial s}\right)_{x^{\alpha}, \rho} & \frac{1}{\rho}\left(\frac{\partial \phi_{j}^{\alpha}}{\partial s}\right)_{x^{\alpha}, \rho} \\
\left(\frac{\partial p}{\partial \nabla_{i} x^{\beta}}\right)_{s, \rho} & \left(\frac{\partial T}{\partial \nabla_{i} x^{\beta}}\right)_{s, \rho} & \frac{1}{\rho}\left(\frac{\partial \phi_{j}^{\alpha}}{\partial \nabla_{i} x^{\beta}}\right)_{s, \rho}
\end{array}\right] .
$$

Although there are only $n+2$ independent hydrodynamic variables this matrix is a square matrix of dimension $3 n+2$. This difference should be kept in mind and we shall return to it later. It reflects the interdependence of the variations $\nabla_{i} x^{\beta}$ and $\nabla_{j} x^{\beta}(i \neq j)$. Indeed, since some of these derivatives may vanish, the $(3 n+2) \times(3 n+2)$ matrix in Eq. (2.21) may not have an inverse. For determining conditions of stability it is necessary to consider the matrix which describes the independent $(n+2)$ degrees of freedom $x^{\beta}$ and the forces associated with deviations in them. Note also that although $\phi_{i}{ }^{\alpha}$ is a small quantity (resulting from expansion about equilibrium), $\left(\partial \phi_{i}{ }^{\alpha} / \partial \rho\right)$ need not be, that is, $\phi_{i}{ }^{\alpha} \sim \delta \rho$ and $\left(\partial \phi_{i}{ }^{\alpha} / \partial \rho\right)=\rho(\partial / \partial \rho)\left(\phi_{i}{ }^{\alpha} / \rho\right)$.

If we could neglect dissipative effects completely, that is, if $R$ in Eq. (2.11) could be taken to be zero, all of the remaining hydrodynamic fluctuations could be taken at constant $s$. Thus the equations of motion and the characteristic frequencies can all be expressed in terms of the isentropic derivatives in the upper left-hand part of Eqs. (2.21). This is not the case for the mode intensities (the mean-square fluctuations in the amplitudes of the hydrodynamic variables). They are more simply related to the isothermal derivatives. Hence, even when dissipation is omitted, the full matrix (2.21) is required for a complete hydrody namic theory.

Before going on to consider specifically the various liquid crystalline and crystalline cases, it may be in order to make another remark on the question of a symmetric versus an unsymmetric stress tensor. In the present discussion, we never obtained a form for the reversible part of the stress tensor; we only obtained an expression for the reversible part of $\nabla_{i} \sigma_{j i}$. Any stress tensor that leads to this expression for the acceleration is satisfactory. It is certainly possible to use an unsymmetric one, but a symmetric one can always be constructed and employed to advantage. Moreover, with a symmetric one, the effects of the boundary are described as taking place at the boundary and not transformed from the surface to the volume. The rearrangement is hardly startling since one such tensor is the averaged microscopic stress tensor, and in the microscopic theory there is no place for "volume torques" despite the fact that by exerting the proper forces on the boundaries we can produce a different local configuration from the one that would result with free surfaces.

We can also illustrate that it is possible to construct a macroscopic stress tensor which is symmetric in another fashion-a fashion which will clarify the connection between our discussion and the conventional elastic theory of solids. It is sufficient to consider the dissipation-free limit. The reactive effects, including the reactive part of the stress tensor determined in this limit, are not modified by dissipation because in the limit $q \rightarrow 0$ the irreversible part of the hydrodynamic motions is negligible. Because the dissipative terms have time-reversal properties opposite to the reactive terms they are orthogonal to first order. If we also assume that there is only one variable of the type $\left\{x^{\alpha}\right\}$ satisiying $\dot{x}=v_{3}+$ (higher-order terms), and use Eq. (2.18),

$$
\rho \dot{v}_{i}=-\nabla_{i} p+\delta_{i 3}\left(\nabla_{j} \phi_{j}\right),
$$


with the thermodynamic identities that follow from Eq. (2.21), we find

$$
\begin{aligned}
\rho \ddot{v}_{i}= & \nabla_{i}\left[\left(\frac{\partial p}{\partial \rho}\right) \rho \nabla_{k} v_{k}-\rho\left(\frac{\partial \phi_{j}}{\partial \rho}\right) \nabla_{j} v_{3}\right] \\
& -\delta_{i 3} \nabla_{j}\left[\rho\left(\frac{\partial \phi_{j}}{\partial \rho}\right) \nabla_{k} v_{k}-\left(\frac{\partial \phi_{j}}{\partial \nabla_{k} x}\right) \nabla_{k} v_{3}\right] .
\end{aligned}
$$

Terms of order $v^{2}$ have been neglected.

Multiplying by $\dot{v}_{i}$ and integrating over space, neglecting surface terms, we obtain

$$
\begin{aligned}
& \frac{d}{d t} \int d \overrightarrow{\mathrm{r}}\left[\frac{1}{2}|\dot{\overrightarrow{\mathrm{v}}}|^{2}+\frac{1}{2}\left(\frac{\partial p}{\partial \rho}\right)\left(\nabla_{i} v_{i}\right)\left(\nabla_{j} v_{j}\right)\right. \\
& \left.+\frac{1}{2 \rho}\left(\frac{\partial \phi_{k}}{\partial \nabla_{j} x}\right)\left(\nabla_{j} v_{3}\right)\left(\nabla_{k} v_{3}\right)-\left(\frac{\partial \phi_{j}}{\partial \rho}\right)\left(\nabla_{j} v_{3}\right)\left(\nabla_{i} v_{i}\right)\right]=0 .
\end{aligned}
$$

For the dissipation-free case we may always consider stationary oscillatory states of a single defined frequency $\omega$. In that case we may divide Eq. (2.24) by $\omega^{2}$ and the integrand will be exactly equivalent to the expression for the elastic energy employed in the conventional elastic theories of solids. Anticipating the discussion following (3.2) regarding the long-wavelength dependence of $\partial \phi_{k} /$ $\partial \nabla_{j} x$, when $j, k=1,2$ as in the case of solids, (2.24) can be entirely written in terms of the "deformation variables" $\left(\nabla_{j} v_{k}+\nabla_{k} v_{j}\right)$ by partial integration. Using identities of the type

$$
\begin{aligned}
2 \nabla_{j} \nabla_{k} v_{l}=\nabla_{j}\left(\nabla_{k} v_{t}+\nabla_{l} v_{k}\right)+\nabla_{k}\left(\nabla_{j} v_{l}+\nabla_{l} v_{j}\right) \\
-\nabla_{l}\left(\nabla_{j} v_{k}+\nabla_{k} v_{j}\right)
\end{aligned}
$$

this can be shown to be true for terms of all orders in the wave vector. It follows that a symmetric elastic stress tensor (in particular the derivative of the energy obtained above), can always be found. More important, the connection between an elastic description and a fluid description is clarified. When terms proportional to the second term in (2.16) are necessary, second-order elastic constants result. It is this description which was utilized in Martin et al. (MPS) ${ }^{11}$ and it is completely equivalent to the one employed here.

Note also that, as in a solid, there is a stability condition associated with a nonsingular upper $2 \times 2$ part of the matrix (2.21). Taking $x^{\alpha}$ to have the symmetry of the 3 component of a vector, i.e., $x_{i}^{\alpha}=x \delta_{i 3}$, we may write

$$
\begin{aligned}
& \left(\frac{\partial p}{\partial \rho}\right)_{s, x}=\frac{1}{\rho^{2}}\left(\frac{\partial^{2}(\epsilon / \rho)}{\partial(1 / \rho) \partial(1 / \rho)}\right)_{s, x} \geq 0, \\
& \frac{1}{\rho}\left(\frac{\partial \phi_{3}}{\partial \nabla_{3} x}\right)_{s, \rho}=\frac{1}{\rho}\left(\frac{\partial^{2}(\epsilon / \rho)}{\left(\partial \nabla_{3} x\right)\left(\partial \nabla_{3} x\right)}\right)_{s, \rho} \geq 0, \\
& \left(\frac{\partial p}{\partial \nabla x}\right)_{s, \rho}=\rho\left(\frac{\partial \phi_{3}}{\partial \rho}\right)_{s, x}=-\frac{\partial^{2}(\epsilon / \rho)}{\partial\left(\nabla_{3} x\right) \partial(1 / \rho)},
\end{aligned}
$$

and, for the special case that spatial gradients are along the 3 direction,

$$
\frac{1}{\rho}\left(\frac{\partial p}{\partial \rho}\right)_{x}\left(\frac{\partial \phi_{3}}{\partial \nabla_{3} x}\right)_{\rho}-\left(\frac{\partial \phi_{3}}{\partial \rho}\right)_{x}^{2} \geq 0 .
$$

The stability criterion for arbitrary spatial gradients is more complicated and illustrates the remark made following Eqs. (2.21). For example, as we will discuss below, in the case of smectic $A$-phase liquid crystals we shall also require higher-order terms since $\left(1 / \nabla^{2}\right)\left(\partial \nabla_{1} \phi / \partial x\right)$ vanishes in the state of homogeneous thermal equilibrium in the absence of externally applied forces. On the other hand, if we carred out a microscopic calculation for $\ddot{v}_{i}(\overrightarrow{\mathrm{q}})$, where $v_{i}(\overrightarrow{\mathrm{q}})$ is the spatial Fourier transform (wave vector $\vec{q}$ ) of the microscopic quantity $\rho^{-1} g_{i}(\vec{r})$, we would not only find the terms that appear in Eq. (2.23) but higher-order terms proportional, for example, to $\nabla_{i} \nabla_{j} \nabla_{k} \nabla_{l} v_{m}$. As we mentioned above, when the lower-order terms that appear explicitly in Eq. (2.23) vanish, these higher-order terms must be included in the elastohydrodynamic theory. Although there are several ways to account for them, probably the simplest is to carry out the previous discussion in terms of the spatial Fourier transforms of the hydrodynamic quantities $\delta \rho(\overrightarrow{\mathrm{q}} t), \delta v_{i}(\overrightarrow{\mathrm{q}} t), \delta \in(\overrightarrow{\mathrm{q}} t), \delta x^{\alpha}(\overrightarrow{\mathrm{q}} t)$, etc. In place of the operator $\nabla_{f}$, we then have the algebraic quantity $i q_{j}$.

To illustrate the conditions leading to higherorder elastic constants consider a nematic phase, in which the extra hydrodynamic variables associated with the broken symmetry are angles rather than displacements. The variables $\left\{x^{\alpha}\right\}$ are then two independent director components $\delta n_{1}$ and $\delta n_{2}$. In the dissipation-free region they are related to $\vec{v}$ by Eq. (2.17). The thermodynamic derivative in Eq. (2.18) thus has the formal property

$$
\left(\frac{\partial \dot{n}_{i}}{\partial v_{j}}\right)_{T, p, \phi}=\mu_{i j k} \nabla_{k} \text {. }
$$

The interpretation of Eq. (2.27) is that if $n_{i}(\overrightarrow{\mathrm{r}})$ and $v_{j}(\overrightarrow{\mathrm{r}})$ are replaced by $n_{i}(\overrightarrow{\mathrm{q}})$ and $v_{j}(\overrightarrow{\mathrm{q}})$, then the equation is satisfied when $\nabla_{k}$ is replaced by $i q_{k}$. In this same sense, whenever the lower-order thermodynamic derivatives in (2.21) vanish, we must consider higher-order terms. With proper account for the difference between variables and their complex conjugates, the cross-derivative relation (2.18) becomes

$$
\left(\frac{\partial \dot{n}_{i}}{\partial v_{j}}\right)_{T, p, \phi}=\mu_{i j k} \nabla_{k}=-\left(\frac{\partial \dot{g}_{j}}{\partial\left(\nabla_{k} \phi_{k}^{i}\right)}\right)_{T, \phi, \overrightarrow{\mathrm{v}}} .
$$

We also have in the nematic phase terms like

$$
\rho\left(\frac{\partial \phi_{j}^{i}}{\partial \rho}\right)_{s, n}=\nu_{i j k} \nabla_{k}=-\left(\frac{\partial p}{\partial\left(\nabla_{j} n_{i}\right)}\right)_{\rho, s}
$$

and others coupling $T$ to $\nabla n$ in the matrix (2.21). In place of Eq. (2.23), when dissipative effects are neglected, we obtain for a nematic phase 


$$
\begin{aligned}
\rho \ddot{v}_{i}= & \rho\left(\frac{\partial p}{\partial \rho}\right)_{s, n} \nabla_{i} \nabla_{j} v_{j} \\
& +\nu_{k j t} \mu_{k s t} \nabla_{l} \nabla_{i} \nabla_{j} \nabla_{t} v_{s}+\mu_{j i r} \nu_{j k s} \nabla_{r} \nabla_{k} \nabla_{s} \nabla_{t} v_{t} \\
& -\mu_{j i r}\left(\frac{\partial \phi_{k}^{j}}{\partial\left(\nabla_{l} n_{m}\right)}\right)_{s, \rho} \mu_{m s t} \nabla_{r} \nabla_{k} \nabla_{l} \nabla_{t} v_{s} .
\end{aligned}
$$

The transverse modes are characterized by secondorder elastic constants. The terms $\left[\partial \phi_{k}{ }^{j}\right]$ $\left.\partial\left(\nabla_{l} n_{m}\right)\right]_{s, \rho}$ are the "usual" isentropic Frank elastic constants. The $\{\nu\}$ terms couple density to order-parameter fluctuations. While it appears as if five independent second-order elastic constants are necessary in general to specify the hydrodynamic frequencies in a uniaxial nematic shase, only four are really required. The term that arises from $\left(\partial \phi_{k}{ }^{j} / \partial \rho\right) \dot{\rho}$ represents a negligible correction to the term $(\partial p / \partial \rho) \dot{\rho}$ because the former always appears in combination with the latter and contributes in Eq. (2.30) a term of order $\nabla^{4}$ (i.e. , of the order of the Frank energy, not $\nabla^{2}$ ). Since it is always possible to redefine the pressure by a term of order $\nabla^{2}$ [and thus to eliminate this term in favor of a term of order $\nabla^{6}$, by completing the square in the expression for the elastic energy analogous to (2.24) that is obtained with the aid of (2.30)], $\nu_{i j k}$ does not play a role. The connection between the remaining four "elastic" parameters and the three Frank constants and the parameter $\lambda$ [i.e., the quantity $\left(\partial \sigma_{i j} / \partial \phi^{k}\right)_{\rho}$ which describes the stress induced by a static change of magnetic field in a nematic liquid crystal and is analogous to the quantity $(d p / d T)_{\rho}$ in a simple fluid] is given in FLMPS. The four parameters occur in a similar fashion in this adiabatic-elasticity-theory version, whereas the three Frank elastic parameter $\mathrm{s}^{21}$ and the reactive-flowreorientation-variable coupling appear in a rather different fashion in the director formulation. ${ }^{22}$

\section{CHOLESTERIC AND SMECTIC $\boldsymbol{A}$ LIQUID CRYSTALS}

The symmetry property that distinguishes a smectic $A$ liquid crystal from the isotropic fluid is the fact that although it is translationally invariant to arbitrary displacements in two orthogonal spatial directions, it is not translationally invariant to displacements in the 3 direction. Thus the extra, slow, independent hydrodynamic variable for a smectic $A$ phase describes the displacement of the smectic layers in this third direction. Note that it is the local displacement of the layers, not the angular rotation of the 3 direction, that is used. Local homogeneous rotations of a layered structure produce infinite displacements of the layered pattern at infinite distances from the rotation axis, and this is not consistent with the local hydrodynamic theory of small fluctuations. Also, since inhomogeneous variations in layer displacement induce local rotations, the two types of deformations (rotations and displacements) are not completely independent. There is only one hydrodynamic variable, the layer displacement in the 3 direction. If we call it $x$, we arrive at the following equations of motion:

$$
\begin{aligned}
& \dot{\rho}+\rho \nabla_{i} v_{i}=0, \\
& \dot{x}-v_{3}=\zeta \nabla_{i} \phi_{i}+\xi T^{-1} \nabla_{3} T, \\
& \dot{Q}=\dot{\epsilon}-\rho^{-1}(\epsilon+p) \dot{\rho}=\xi \nabla_{3} \nabla_{i} \phi_{i}+\kappa_{\perp} \nabla^{2} T+\left(\kappa_{11}-\kappa_{\perp}\right) \nabla_{3}^{2} T, \\
& \rho \dot{v}_{i}=-\nabla_{i} p+\delta_{i 3} \nabla_{j} \phi_{j}+\eta_{i j k l} \nabla_{j} \nabla_{l} v_{k},
\end{aligned}
$$

which, together with the thermodynamic parameters that relate $\phi, T, p$, to $x, Q$ (or $\epsilon$ ), and $\rho$, determine the properties of the system. The thermodynamic parameters are given by the symmetric positive definite matrix ${ }^{23}$

$$
\left[\begin{array}{cccc}
\rho^{2}\left(\frac{\partial p}{\partial \rho}\right)_{s, x} & \rho^{2}\left(\frac{\partial T}{\partial \rho}\right)_{s, x} & \rho\left(\frac{\partial \phi_{3}}{\partial \rho}\right)_{s, x} & 0 \\
\left(\frac{\partial p}{\partial s}\right)_{x, \rho} & \left(\frac{\partial T}{\partial s}\right)_{x, \rho} & \frac{1}{\rho}\left(\frac{\partial \phi_{3}}{\partial s}\right)_{x, \rho} & 0 \\
\left(\frac{\partial p}{\partial \nabla_{3} x}\right)_{s, \rho} & \left(\frac{\partial T}{\partial \nabla_{3} x}\right)_{s, \rho} & \frac{1}{\rho}\left(\frac{\partial \phi_{3}}{\partial \nabla_{3} x}\right)_{s, \rho} & 0 \\
0 & 0 & 0 & \frac{1}{\rho}\left(\frac{\partial \phi_{1}}{\partial \nabla_{1} x}\right)_{s, \rho}
\end{array}\right]
$$

We take the gradient in the 1-3 plane since all directions in the 1-2 plane are equivalent. The zeros in (3.2) correspond to thermodynamic derivatives of $\phi_{1}=\left[\partial(\epsilon / \rho) / \partial\left(\nabla_{1} x\right)\right]_{s, \rho}$, and by symmetry must be proportional to at least one power of $\nabla_{1}$ and vanish in the limit $q \rightarrow 0$. Thus, in accordance with our earlier discussion of higher-order elastic constants, these terms can be disregarded since lower-order terms always dominate them. Note also that in smectic $A$ liquid crystals there is no restoring force to either a uniform shear produced by sliding layers over each other or to a 
uniform rotation about an axis in the plane of the layers. This is a consequence of the fact $t$ lat there is no marking to describe motions along the layers; i.e., the translational symmetry in these directions is unbroken. ${ }^{4}$ Since the effective elastic energy [Eq. (2.24)] can always be expressed in terms of symmetric strains $\left(\partial_{3} v_{1}+\partial_{1} v_{3}\right)$, the absence of elastic restoring forces to both uniform rotations $\left(\partial_{3} v_{1}-\partial_{1} v_{3}\right)$ and shears $\left(\partial_{3} v_{1}\right)$ implies the absence of resistance to a uniform deformation $\left(\partial_{1} v_{3}\right)$. Thus the elastic energy associated with $\left(\partial_{3} v_{1}+\partial_{1} v_{3}\right)$, that is, $\left(\partial \phi_{1} / \partial \nabla_{1} x\right)_{\rho, s}$, is of higher order in $q$ than conventional elastic energies. The lowest nonvanishing terms permitted by symmetry are of order $\nabla_{1}^{2}$ or $\nabla_{3}^{2}$. Whenever $\nabla_{3} \neq 0$ (i.e., $q_{3} \neq 0$ ) energies related to $\phi_{1}$ are of higher order, and can be neglected in comparison to other terms in (3.2). If $\nabla_{3}=0$ (i. e., $\left.q_{3}=0\right)$ the term in $\left(\partial \phi_{1} /\right.$ $\left.\partial \nabla_{1} x\right)_{\rho, s}$ proportional to $\nabla_{1}^{2}$ must be considered. There are therefore six first-order "elastic" constants in a smectic $A$ phase, corresponding to the upper $3 \times 3$ block of the symmetric matrix, (3.2), and one second-order constant,

$$
\left(\frac{\partial \phi_{1}}{\partial \nabla_{1} x}\right)_{p, T}=\left(\frac{\partial \phi_{1}}{\partial \nabla_{1} x}\right)_{\rho, s}=-K \nabla_{1}^{2} .
$$

In accordance with Eqs. (2.15) and (3.1), the dissipative parameters are described by one $4 \times 4$ positive definite matrix coupling $\nabla_{i} \phi_{i}$, the three components of $\nabla_{i} T$, and the $6 \times 6$ positive definite matrix for the viscosities. The symmetry of the problem reduces the $4 \times 4$ matrix to the four constants $\kappa_{\|}, \kappa_{\perp}, \xi$, and $\zeta$ satisfying

$$
\kappa_{\perp} \geq 0, \quad \kappa_{11} \geq 0, \quad \zeta \geq 0, \quad \kappa_{\|} \zeta-\left(\xi^{2} / T\right) \geq 0,
$$

and to the five viscosities, characteristic of an arbitrary uniaxial system. If $v_{i j} \equiv \frac{1}{2}\left(\nabla_{i} v_{j}+\nabla_{j} v_{i}\right)$, the dissipative part of the stress tensor defining the viscosities $^{24}$ is

$$
\begin{aligned}
& \left(\sigma_{i j}\right)^{D}=-2 \eta_{2} v_{i j}-2\left(\eta_{3}-\eta_{2}\right)\left(v_{i 3} \delta_{j 3}+v_{j 3} \delta_{i 3}\right) \\
& -\left(\eta_{4}-\eta_{2}\right) \delta_{i j} v_{k k}-\left(\eta_{1}+\eta_{2}-4 \eta_{3}-2 \eta_{5}+\eta_{4}\right) \delta_{i 3} \delta_{j 3} v_{33} \\
& \quad-\left(\eta_{5}-\eta_{4}+\eta_{2}\right)\left(\delta_{i j} v_{33}+\delta_{i 3} \delta_{j 3} v_{k k}\right),
\end{aligned}
$$

and it must satisfy the conditions

$\eta_{1} \eta_{4} \geq \eta_{5}^{2}, \quad \eta_{2} \geq 0, \quad \eta_{3} \geq 0, \quad \eta_{4} \geq 0, \quad \eta_{1} \geq 0$.

The discussion of the modes is simplest when $\vec{\nabla}$ (or $\vec{q}$ ) is in the 3 direction. The two equations for the transverse velocity are decoupled from one another and from the other four equations of motion. The frequency for these two modes, taking $q \equiv q_{3}$, is

$$
\omega=-i \eta_{3} q^{2} \rho^{-1} \text {. }
$$

The remaining four equations become [with $\delta Q$ $\left.\equiv \delta \epsilon-\rho^{-1}(\epsilon+p) \delta \rho\right]$

$$
-i \omega \delta \rho+i \rho q v_{3}=0 \text {, }
$$

$$
\begin{aligned}
& -i \omega \delta Q+\xi q^{2} \delta \phi_{3}+\kappa_{\|} q^{2} \delta T=0 \\
& -i \omega \delta x-v_{3}-i \zeta q \delta \phi_{3}-i(\xi / T) q \delta T=0 \\
& -i \omega \rho v_{3}+i q\left(\delta p-\delta \phi_{3}\right)+\eta_{1} q^{2} v_{3}=0 \\
& -i \omega \delta\left(\nabla_{3} x\right)-i \omega \rho^{-1} \delta \rho+\zeta q^{2} \delta \phi_{3}+(\xi / T) q^{2} \delta T=0 \\
& -i \omega \delta Q+\xi q^{2} \delta \phi_{3}+\kappa_{\|} q^{2} \delta T=0 \\
& \omega^{2} \delta \rho-q^{2}\left(\delta p-\delta \phi_{3}\right)+i \eta_{1} q^{2} \omega \rho^{-1} \delta \rho=0
\end{aligned}
$$$$
\text { or }
$$

By changing the notation in Eqs. (3.9) we could make them identical to the equations that describe the coupling between sound propagation, thermal conductivity, and diffusion in a two-component fluid. The quantity $\nabla_{3} x$ would play the role of the concentration fluctuations and $\phi_{3}$ the role of the osmotic pressure. ${ }^{8}$ Although it is somewhat complicated, this mode structure of binary fluids has been discussed extensively. We can therefore immediately give the smectic-phase modes when $\vec{q}$ is in the 3 direction. In addition to the two shear waves, there is a sound wave whose attenuation depends on all of the transport coefficients and thermodynamic derivatives; its velocity is given simply by

$$
c^{2}=\left(\frac{\partial p}{\partial \rho}\right)_{s, x}-2\left(\frac{\partial \phi_{3}}{\partial \rho}\right)_{s, x}+\frac{1}{\rho}\left(\frac{\partial \phi_{3}}{\partial \nabla_{3} x}\right)_{\rho, s} .
$$

There are also two diffusive modes whose frequencies depend on the transport coefficients $\xi$, $\kappa_{11}, \zeta$, and three thermodynamic derivatives. A light-scattering experiment in which the momentum transfer is along the 3 direction would observe the usual Brillouin peaks and two superposed central peaks. The relative intensities of the various contributions would depend on the coupling between $\delta \epsilon$ and $\delta T, \delta \rho$, and $\partial_{3} \delta x$. It would be interesting to measure the analogies to thermal and particle diffusion.

When $\vec{\nabla}$ (or $\vec{q}$ ) is in a direction perpendicular to the 3 direction, say the 1 direction, there is one decoupled shear wave, $v_{2}$, with frequency $\omega$ $=-i n_{2} q^{2} / \rho$; three coupled modes, $v_{1}, \delta \rho$, $\delta T$, which yield sound waves and thermal diffusion as in a normal fluid; and two others, reminiscent of the nematic phase, coupling $v_{3}$ and $\partial_{1} x$. The latter plays the same role as $\delta n_{1}$ in a nematic liquid crystal. The frequencies of the last pair of modes are given approximately, as in the nematic phase, by

$$
\omega_{f} \sim-i \eta_{3} q^{2} \rho^{-1}, \quad \omega_{s} \sim-i K q^{2} / \eta_{3}
$$

Note that these two frequencies are determined solely in terms of the viscosity. In this case $\zeta$ does not contribute and the result is therefore the same as that obtained by MPS when the additional dissipative parameter akin to $\zeta$ was incorrectly omitted. We shall not discuss the Landau-Placzek spectrum 
beyond noting that in the standard expression ${ }^{1}$ for it, the thermal conductivity is $\kappa_{\perp}$ and the viscosity $\eta_{2}+\eta_{4}$

The additional transport parameters $\zeta$ and $\xi$ actually play no role in the case in which $\vec{\nabla}$ (or $\overrightarrow{\mathrm{q}}$ ) is perpendicular to the 3 direction, and the higherorder elastic term only plays a role in this direction. More quantitatively it is important only when $q_{1}{ }^{4} / q_{3}{ }^{2}>\left(\partial \phi_{3} / \partial \nabla_{3} x\right) \eta^{-2}$. The inequality gives the condition under which the coupled shear orderparameter fluctuations change from the form described by Eqs. (3.11) to propagating modes.

For arbitrary direction of $\vec{\nabla}$ (or $\vec{q}$ ) there are five coupled equations. They could be solved, but the result would not be very illuminating. It seems more worthwhile and informative to determine the qualitative behavior of the solution from Eqs. (2.23) which applies in the dissipation-free limit. In this limit the coupling between $v_{1}$ and $v_{3}$ leads to a quadratic equation in the variable $\omega^{2}$,

$$
\begin{aligned}
& \omega^{4}-\omega^{2}\left\{\left(\frac{\partial p}{\partial \rho}\right)_{s, x}\left(q_{1}^{2}+q_{3}^{2}\right)\right. \\
& \left.+\left[\frac{1}{\rho}\left(\frac{\partial \phi_{3}}{\partial \nabla_{3} x}\right)_{s, \rho}-2\left(\frac{\partial \phi_{3}}{\partial \rho}\right)_{s, x}\right] q_{3}{ }^{2}+K q_{1}{ }^{4}\right\} \\
& +\left[\frac{1}{\rho}\left(\frac{\partial p}{\partial \rho}\right)_{s, x}\left(\frac{\partial \phi_{3}}{\partial \nabla_{3} x}\right)_{s, \rho}-\left(\frac{\partial \phi_{3}}{\partial \rho}\right)_{s, x}^{2}\right] q_{1}^{2} q_{3}^{2}=0 \text {. }
\end{aligned}
$$

The solution of Eq. (3.12) yields two propagating modes with velocitites $c_{1}$ and $c_{2}$ satisfying (to lowest order in $q$ )

$$
\begin{aligned}
& c_{1}^{2}+c_{2}^{2}=\left(\frac{\partial p}{\partial \rho}\right)_{s, x}+\cos ^{2} \psi\left[\frac{1}{\rho}\left(\frac{\partial \phi_{3}}{\partial \nabla_{3} x}\right)_{s, \rho}-2\left(\frac{\partial \phi_{3}}{\partial \rho}\right)_{s, x}\right], \\
& c_{1}^{2} c_{2}^{2}=\cos ^{2} \psi \sin ^{2} \psi\left[\frac{1}{\rho}\left(\frac{\partial p}{\partial \rho}\right)_{s, x}\left(\frac{\partial \phi_{3}}{\partial \nabla_{3} x}\right)_{s, \rho}-\left(\frac{\partial \phi_{3}}{\partial \rho}\right)_{s, x}^{2}\right],
\end{aligned}
$$

where $\psi$ is the angle between the direction of $\vec{\nabla}$, or $\overrightarrow{\mathrm{q}}$, and the symmetry axis 3 . Thus, in a smectic $A$ phase, neglecting dissipation, we obtain two sound waves, with speeds determined by Eq.

(3.13). In addition to these four modes, there are two of zero frequency. One of them corresponds to the shearing motion $\partial_{3} v_{2}$ (or $\partial_{1} v_{2}$ ) for which there is no associated elastic energy and the other is the thermal-conductivity mode which, in the absence of dissipation, always has zero frequency. The effect of including dissipation is to make the zerofrequency shear mode $\left(\partial_{3} v_{2}\right)$ diffusive with a frequency [Eqs. (3.7), for example] determined solely by the viscosity coefficients, to make the zerofrequency thermal-conduction mode diffusive with a frequency which is an involved function of the dissipative parameters, and to add attenuation to the propagating modes. The attenuation coefficients will be frightening algebraic functions of the dissipative and thermodynamic parameters. ${ }^{25}$

The results are illustrated in Figs. 1 and 2 .
Figure 1 shows the velocity of the two propagating waves as a function of orientation relative to the symmetry axis of the smectic $A$ phase. Figure 2 (a) shows the spectral density for a general hydrodynamic variable, at arbitrary $\vec{q}$, as a function of $\omega$. There are two Brillouin peaks, $\omega^{2}=c_{1}^{2} q^{2}$ and $\omega^{2}=c_{2}{ }^{2} q^{2}$, and a central peak that consists of the thermal-diffusion mode and the shear velocity $v_{2}$ mode. Thus, in general, the central peak is the sum of two Lorentzians. On the other hand, in an experimental measurement, such as polarized light scattering, the coupling to $v_{2}$ may vanish. When it does the central peak is essentially a single Lorentzian. Figure 2(b) sketches the same effects for $\vec{q}$ in the 3 direction. There is only one soundlike mode and the central peak is the superposition of four Lorentzian lines. Again, if in a given experiment the coupling to the diffusive shear velocity modes is absent, the central peak will consist of two superposed Lorentzians, in analogy with the two-component fluid. For $\overrightarrow{\mathrm{q}}$ in the 1 direction, Fig. 2(c), the mode structure reduces to a LandauPlaczek triplet superposed on a central peak that consists of the coupled nematiclike modes $\partial_{1} x$ and $\partial_{1} v_{3}$ (shown separately in the figure). While the $\partial_{1} v_{2}$ mode can also contribute to the central peak, it often does not couple to experimentally observed variables for the reasons mentioned above.

The theory described for a smectic $A$ phase is also applicable to cholesteric liquid crystals and its essential results are identical to the theory described by Lubensky. ${ }^{5}$ From a macroscopic point of view the only difference between the symmetry of cholesteric and smectic $A$ liquid crystals is that the former lacks a mirror symmetry that is present for the latter. The presence, or ab-

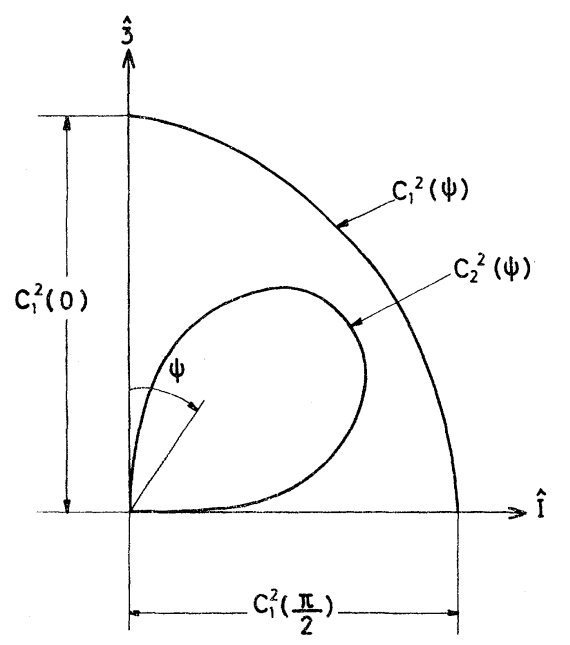

FIG. 1. Speed of propagating modes in a smectic $A$ phase as a function of direction of propagation. 
Modes that couple to density

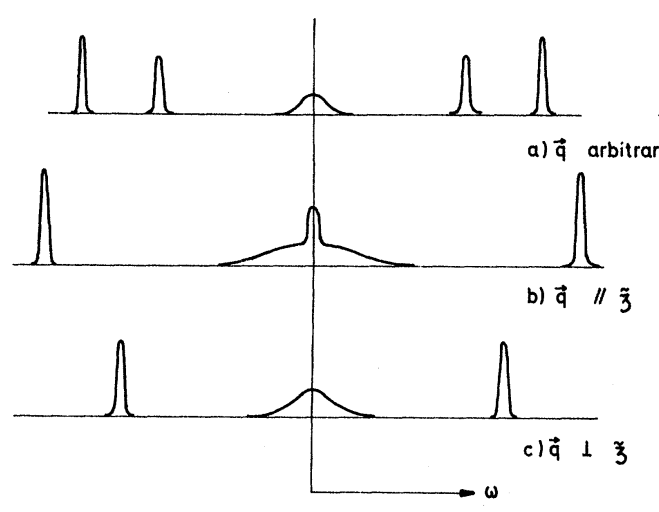

Modes that do not couple to density

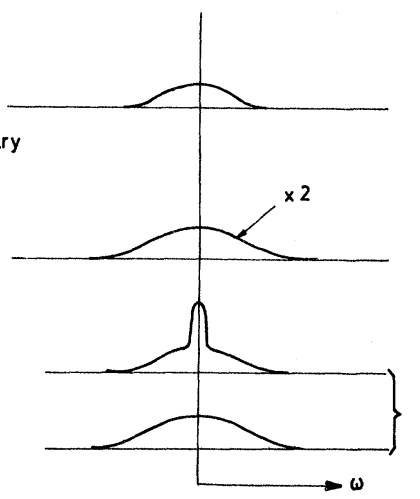

FIG. 2. Hydrodynamic spectral densities for a smectic $A$ phase with $\overrightarrow{\mathrm{q}}$ along different directions. For $\overrightarrow{\mathrm{q}}$ $\| \hat{3}$, the central line that couples to the density is the sum of two Lorentzians that in general have widely different half-widths. The part of the spectral density that does not couple to the density consists of two Lorentzians of identical half-widths. They have different polarizations and do not couple. For $\vec{q} \perp \hat{3}$ the part that does not couple to the densities consists of one pair of Lorentzians that has different symmetry, and thus does not couple to a third Lorentzian. For all orientations of $\overrightarrow{\mathrm{q}}$, there are six modes. sence, of this symmetry element changes absolutely nothing in the analysis and the hydrodynamic theories of the two systems are identical. Of course, as presented here, hydrodynamics is rigorously applicable only in the limit of $(\omega, \vec{q}) \rightarrow 0$. Thus a very real difference between the two systems will be the magnitude of $\omega$ and $q$ that can be considered small enough to apply the hydrodynamic theory. A reasonable guess might be $q \ll \pi / d$, where $d$ is either the period of the cholesteric-phase twist or the smectic-phase layer spacing. The hydrodynamic theory is therefore restricted to much smaller wave vectors in cholesteric than in smectic liquid crystals. Likewise, we would expect the various elastic constants and transport parameters to be much smaller in cholesteric than in smectic liquid crystals. In other words, although the theory of this section applies to the long-wavelength low-frequency properties of cholesteric liquid crystals, it is far from clear that it is sufficient for cholesteric liquid crystals at the wavelengths and frequencies of practical interest in light scattering. Note also that when $q$ $\rightarrow 0$ and $\delta x$ is constant the deformation is a uniform rotation of the molecular orientations about the cholesteric axis (i.e., the 3 direction). The physical effects resulting from the coupling of this deformation to a temperature gradient $\partial_{3} T$ and similar couplings can be discussed with the aid of Eqs. (3.8). The relation between these equations and the description of a two-component fluid is of some assistance in understanding the type of effects that might be expected in a cholesteric phase. We know that in a two-component fluid, it is boundary conditions that determine whether or not there is particle diffusion and thermal conduction or only the latter. Likewise it is boundary conditions that are crucial for determining whether a temperature gradient will induce a uniform $\dot{x}$ in the cholesteric phase. $^{26}$

\section{SMECTIC $C$ LIQUID CRYSTALS}

From a macroscopic point of view, smectic $C$ liquid crystals differ from smectic $A$ liquid crystals in only one respect. ${ }^{27}$ Whereas the smectic $A$ phase is invariant under all rotations about the 3 axis (we take the 3 axis to be the single direction in which the smectic $A$ phase is not translationally invariant), a smectic $C$ phase is not. For a smectic $C$ phase we can again take the 3 direction perpendicular to the layers and define a second direction, the 1 direction, by the intersection of the layers and the mirror planes. This identification of the 1-3 plane is consistent with the microscopic model of a smectic $C$ phase in which one imagines molecules tilted from the 3 direction towards the 1 direction. Thus, we identify two "extra" hydrodynamic variables for a smectic $C$ phase. One, as in a smectic $A$ phase, corresponds to the translation of the smectic liquid-crystal layers. The other, reminiscent of the nematic phase, corresponds to the rotation of the 1 direction about the 3 axis. Taking the displacement variable $x=x^{\alpha}(\alpha=3)$, the rotation variable $\delta n_{2}=x^{\alpha}(\alpha=2)$, and using $\phi_{i}=\phi_{i}{ }^{\alpha}(\alpha=3)$, $h_{i}=\phi_{i}^{\alpha}(\alpha=2)$, Eqs. (2.15), (2.16), (2.18), and (2.28), and considering the constraints of symmetry, we obtain

$$
\begin{aligned}
& \dot{\rho}+\rho \nabla_{i} v_{i}=0, \\
& \dot{x}-v_{3}=\zeta \nabla_{i} \phi_{i}+T^{-1} \xi_{j} \nabla_{j} T, \\
& \dot{n}_{2}-\mu_{2 j k} \partial_{k} v_{j}=\gamma^{-1} \nabla_{i} h_{i}, \\
& \rho \dot{v}_{i}=-\nabla_{i} p+\delta_{i 3} \nabla_{j} \phi_{j}-\mu_{2 i k} \nabla_{k} \nabla_{j} h_{j}+\eta_{i j k l} \nabla_{j} \nabla_{l} v_{k}, \\
& \dot{Q} \equiv \dot{\epsilon}-\rho^{-1}(\epsilon+p) \dot{\rho}=\kappa_{i j} \nabla_{i} \nabla_{j} T+\xi_{j} \nabla_{j} \nabla_{i} \phi_{i} .
\end{aligned}
$$

There are, in the biaxial smectic $C$ phase, 13 independent viscosity parameters $\eta_{i j k l}$, four thermal-conductivity parameters $\kappa_{i f}$, two parameters $\xi_{i}$, one $\zeta$, and one $\gamma$ for a total of 21 dissipative parameters. ${ }^{28}$ 
The elastic, and other, reversible parameters can be determined by exactly the same arguments as in a smectic $A$ phase. First we note that in a smectic $C$ phase, as in a smectic $A$ phase, the only uniform stresses that produce strains are those directed along the axis normal to the smectic liquid-crystal layers. Consequently, to lowest order in gradients the expression analogous to the elastic energy [see Eq. (2.24)] is the same for smectic $A$ and smectic $C$ phases. A complete description of the reversible properties of a smectic $C$ phase requires the addition of thermalexpansion coefficients and other terms yielding "effective" elastic energies [see Eq. (2.24)] of higher order in the gradients. Although the number of such additional coefficients can be determined, and we shall do so, their enumeration is of secondary importance. The various functions describing mode intensities and frequencies are cumbersome functions of more parameters than we believe it is reasonable to measure. The qualitative features of the mode structure, however, are not complicated and follow rather easily from the same arguments employed for a smectic $A$ phase.

For arbitrary directions of $\vec{q}$, Eqs. (3.1) for a smectic $A$ phase decoupled the transverse shear mode with $v\left(=v_{2}\right)$ parallel to the layer surfaces from the other five variables $\rho, T, v_{1}, v_{3}$, and $x$. These five resulted, for arbitrary directions, in two pairs of propagating sound waves and one diffusive thermal-conductivity mode. Since the speeds of the propagating modes are solely determined by the first-order elastic properties and since these are the same in both smectic $A$ and smectic $C$ phases, the propagating modes will have exactly the same qualitative behavior in both. Quantitative differences will arise in the attenuation coefficients because of the lower symmetry of the viscosity and thermal-conductivity tensors and also in the special directions where the "propagating" shear mode is determined by the second-order elastic constants (i. e., in directions for which it becomes diffusive). The shear wave that is always diffusive ( $v_{2}$ in a smectic $A$ phase) couples to the variable $n$ in a smectic $C$ phase in much the same manner as in a nematic phase. Thus for propagation in the 1-3 plane the shear mode with $\vec{v}$ parallel to the planes of a smectic $C$ phase and the "director" $\overrightarrow{\mathrm{n}}$ couple to form "fast" and "slow" diffusive modes analogous to those that occur in nematic liquid crystal. When the propagation vector does not lie in the 1-3 plane, the corresponding shear mode involves $v_{1}$ and $v_{2}$, not simply $v_{2}$ as in a smectic $A$ phase.

Although it is possible to write down general expressions for all the mode intensities and frequencies it does not seem worth the effort at the present time and we will simply enumerate the number of coefficients of each type that appear in the general theory. The symmetric matrix, analogous to Eq. (3.2), is larger. In the first place, terms like

$$
\frac{\partial^{2}(\epsilon / \rho)}{\partial \nabla_{k} n \nabla_{l} n}=\frac{\partial h_{l}}{\partial \nabla_{k} n}=\frac{\partial h_{k}}{\partial \nabla_{l} n},
$$

corresponding to Frank-like terms, must be added. Saupe has previously pointed out there are four of them. ${ }^{29}$ Off-diagonal elements like $\left(\partial h_{l} / \partial \rho\right),\left(\partial h_{l} /\right.$ $\left.\partial \nabla_{3} x\right), \quad\left(\partial h_{l} / \partial s\right)$ can be neglected; or, from another equivalent point of view, they can be incorporated into the first-order elastic constants. The logic here is similar to that employed in omitting $\nu$-like terms in the nematic equations.

Second, the 1 and 2 directions in a smectic $C$ phase are not equivalent, as they were in a smectic $A$ phase, and, as previously noted by de Gennes, ${ }^{30}$ there are three terms

$$
\frac{\partial^{2}(\epsilon / \rho)}{\partial \nabla_{i} x \partial \nabla_{j} x}=\frac{1}{\rho} \frac{\partial \phi_{i}}{\partial \nabla_{j} x}=\frac{1}{\rho} \frac{\partial \phi_{j}}{\partial \nabla_{i} x}
$$

where $i$ and $j$ are either 1 or 2, which are similar to the smectic $A$-phase terms [Eq. (3.3)] in that they vanish as the wave vector vanishes. These are also Frank-like terms and three constants are required to specify them. Third, there are offdiagonal terms, also like those in Eq. (3.3), that vanish as $q$ vanishes but which are necessary for $\vec{q}$ in the 1-2 plane where the lower-order elastic terms vanish. Thus, as de Gennes previously pointed out, ${ }^{31}$ there are two terms of the type

$$
\frac{\partial^{2}(\epsilon / \rho)}{\partial \nabla_{i} x \partial \nabla_{j} n}=\frac{\partial h_{j}}{\partial \nabla_{i} x}=\frac{\partial \phi_{i}}{\partial \nabla_{j} n}
$$

for $i$ and $j$ in the 1-2 plane that must be included. These are also Frank-like terms, so that we have a total of $(4+3+2)$ nine terms from Eqs. (4.2)(4. 4).

To complete the description of the higher-order elasticity we must add the terms $\mu_{2 j k}$ that relate $\dot{n}$ to $\partial_{k} v_{j}$ [see Eq. (4.1)]. By the same reasoning used in the nematic phase [see the discussion following Eqs. (2.16) and (2.17)], we see that ${ }^{32}$

$$
\begin{aligned}
\mu_{2 j k} \partial_{j} v_{k}=\frac{1}{2}\left(\partial_{1} v_{2}-\partial_{2} v_{1}\right) & +\frac{1}{2} \lambda^{(3)}\left(\partial_{1} v_{2}+\partial_{2} v_{1}\right) \\
& +\frac{1}{2} \lambda^{(1)}\left(\partial_{3} v_{2}+\partial_{2} v_{3}\right) .
\end{aligned}
$$

Consequently there are two additional parameters, bringing the total number of constants necessary to specify the second-order elasticity ${ }^{33}$ of a smectic $C$ phase to 11 .

Away from the special directions, we have as reversible parameters three first-order elastic constants, the four Frank-Saupe constants described by Eq. (4.2), the two coefficients relating $\dot{n}$ to $\nabla_{i} v_{j}[\mathrm{Eq} .(4.5)]$, and the three that describe the effects of temperature variations. 


\section{CRYSTALS AND SMECTIC $B$ LIQUID CRYSTALS}

A far simpler example than the smectic $C$ phase is the crystal. The reason for the simplicity is that there are no vanishing shear moduli in any direction. Thus the theory outlined in Sec. II applies immediately. There are three displacement order parameters, and therefore eight modes in all. ${ }^{15}$ Six of them correspond to sound waves $\omega$ $= \pm c q$, and the remaining two to defect diffusion and thermal conduction.

The dissipative parameters are given by the two symmetrical matrices involving $\eta$ and $(\kappa, \lambda, \zeta)$, that is,

$$
\begin{aligned}
& \sigma_{i j}{ }^{D}=-\eta_{i j k l} \nabla_{l} v_{k}, \\
& \left(J_{i}^{\epsilon}\right)^{D}=-\kappa_{i j} \nabla_{j} \delta T-\xi_{i}^{j} \nabla_{k} \phi_{k}{ }^{j}, \\
& \left(\dot{x}^{i}\right)^{D}=T^{-1} \xi_{j}{ }^{i} \nabla_{j} \delta T+\zeta^{i j} \nabla_{k} \phi_{k}{ }^{j} .
\end{aligned}
$$

There are, in general, 21 elements in each of the symmetrical positive definite $6 \times 6$ matrices that describe the dissipation. In a uniaxial crystal with no preferred sense, these reduce to five viscosities, and two each of $\kappa, \xi$, and $\zeta$. In a cubic crystal there are three $\eta$ and one of each of the other parameters, an honestly manageable number. The reasoning is the same that is normally applied, respectively, to the elastic constants and thermal expansion.

Likewise, the elastic properties are described by the $8 \times 8$ analogs of (3.2),

$$
\left[\begin{array}{ccc}
\rho^{2}\left(\frac{\partial p}{\partial \rho}\right) & \rho^{2}\left(\frac{\partial T}{\partial \rho}\right) & \rho\left(\frac{\partial \phi_{j}{ }^{i}}{\partial \rho}\right) \\
\left(\frac{\partial p}{\partial s}\right) & \left(\frac{\partial T}{\partial s}\right) & \frac{1}{\rho}\left(\frac{\partial \phi_{j}{ }^{i}}{\partial s}\right) \\
\left(\frac{\partial p}{\partial \nabla_{k} x^{l}}\right) & \left(\frac{\partial T}{\partial \nabla_{k} x^{l}}\right) & \frac{1}{\rho}\left(\frac{\partial \phi_{j}{ }^{i}}{\partial \nabla_{k} x^{l}}\right)
\end{array}\right] .
$$

To the required order, the quantities $\phi_{j}{ }^{i}$ can be taken equal to the symmetric stresses, $p=\frac{1}{3} \phi_{i}{ }^{i}$, $\rho=\nabla_{k} x^{k}$. Therefore, there are, in principle, $21+6$ +1 elastic constants, thermal expansions, and specific heats. Again, the number reduces to 5 $+2+1$ in a uniaxial system and to $3+1+1$ in a cubic crystal (the three elastic constants, thermal expansion, and specific heat). We see then that the elastic constants and dissipative coefficients of the liquid crystal can all be obtained by setting terms in the expressions for a crystal equal to zero and retaining higher-order elastic constants exactly as proposed in a previous letter. Ironically, the theory ${ }^{11}$ proposed there was deficient because the theory of the crystal was incomplete in that it omitted the dissipative parameters $\zeta^{i j}$ that allow for the possibility that mass motion is not synonymous with lattice motion.

The most solid of the liquid crystals is a smectic
$B$ liquid crystal. As far as we know, a smectic $B$ liquid crystal is nothing more than a crystal which cannot sustain uniform 1-3 or 2-3 shears. There are, however, distances defined in three directions. If this is the case, a uniaxial smectic $B$ liquid crystal differs from a uniaxial crystal only in having two second-order elastic constants instead of one of its five first-order elastic constants.

Here, too, while the complete determination of all constants is of little interest, a determination of the effect and size of the additional transport processes seems to be a worthwhile endeavor.

\section{SUMMARY AND CONCLUSIONS}

The basic goal of this paper, to present a unified hydrodynamic theory for crystals, ordered fluids, and simple fluids, is summarized by the equations

$$
\begin{aligned}
& \dot{\rho}+\rho \nabla_{i} v_{i}=0, \dot{\epsilon}+\nabla_{i} J_{i}{ }^{\epsilon}=0, \\
& J_{i}{ }^{\epsilon}-\left(\epsilon^{0}+p\right) v_{i}=-\kappa_{i j} \nabla_{j} \delta T-\xi_{i}^{\alpha} \nabla_{j} \phi_{j}^{\alpha}, \\
& \dot{x}^{\beta}-A_{i}{ }^{\beta} v_{i}-A_{i j}{ }^{\beta} \nabla_{j} v_{i}=(1 / T) \xi_{i}{ }^{\beta} \nabla_{i} \delta T+\zeta^{\beta \alpha} \nabla_{j} \phi_{j}{ }^{\alpha}, \\
& \rho \dot{v}_{i}-\left(A_{i}{ }^{\alpha} \nabla_{j} \phi_{j}{ }^{\alpha}-A_{i k}{ }^{\alpha} \nabla_{k} \nabla_{j} \phi_{j}{ }^{\alpha}\right)+\nabla_{i} \delta p=\eta_{i j k l} \nabla_{l} \nabla_{j} v_{k},
\end{aligned}
$$

together with the various thermodynamic derivatives described by Eqs. (2.21). The unity of the description depends on the presence of terms of the type described in Eqs. (3.3).

In Table I we list the number of different coefficients that enter the general theory for different phases. The caption to the table explains the column headings. Note, for example, that the number given for the two dissipative coefficients that do not couple directly to the order parameter (or the extra hydrodynamic modes in the case of a multicomponent liquid), $\eta_{i j k l}$ and $\kappa_{i j}$, is determined solely by the symmetry of the system. For all the otiler columns the entry depends on both the symmetry of the system and the number and type of the extra hydrodynamic variables above the five always present in a simple fluid.

In the case of nematic liquid crystals, these results are identical with those of Forster et al. (FLMPS). ${ }^{9}$ For the incompressible nematic phase, neglecting thermal conductivity, the equations of motion are equivalent to those derived by the Orsay group after they are supplemented by the "Onsager" relation obtained by Parodi. ${ }^{19}$ With this "Onsager" relation and also neglecting an inertial term which leads to additional nonhydrodynamic modes, the equations proposed by Leslie and Ericksen are also equivalent to the present result. ${ }^{20}$ For cholesteric liquid crystals, they are basically in agreement with the work of Lubensky. ${ }^{5}$ The picture for crystals differs from the conventional one in that it recognizes the difference between deviations from equilibrium of the lattice structure and the mass. As a practical matter, the conventional 
TABLE I. Display of the number of parameters that enter the hydrodynamic theories of different systems (Ref. 34). The column labeled $N$ lists the number of independent hydrodynamic variables. The second through fifth columns give the numbers of transport or dissipative parameters of various types. For the viscosity $\eta_{i j k l}$ the first entry gives the general number of viscous coefficients while the second (in parentheses) gives the number that remain if one adds the condition of incompressibility. The last six columns list the number of coefficients required in the nondissipative system. The four columns labeled "Reversible first-order" give the number of independent thermodynamic derivatives [Eq. (2.21)] of zeroth order in the wave vector: in general [column (a)], for the incompressible system but allowing coupling to temperature (b), for the compressible isothermal system (c), and finally, for the incompressible isothermal system (d). The column "Reversible second order" gives the number of second-order elastic constants not masked by first-order terms, and the last column gives the number of parameters required to specifiy the coupling between order parameter and flow.

\begin{tabular}{|c|c|c|c|c|c|c|c|c|c|c|c|}
\hline \multirow[b]{2}{*}{ Phase } & \multirow[b]{2}{*}{$N$} & \multicolumn{4}{|c|}{ Dissipative (transport) } & \multirow[b]{2}{*}{ (a) } & \multicolumn{4}{|c|}{ Reversible } & \multirow[b]{2}{*}{ Flow } \\
\hline & & $\eta_{i j k l}$ & $\kappa_{i j}$ & $\xi_{i}^{\alpha}$ & $\zeta^{\alpha \beta}$ & & $\begin{array}{c}\text { Firs } \\
\text { (b) }\end{array}$ & $\begin{array}{l}\text { der } \\
\text { (c) }\end{array}$ & (d) & Second order & \\
\hline Simple fluid & 5 & $2(1)$ & 1 & 0 & 0 & 3 & 1 & 1 & 0 & 0 & 0 \\
\hline Binary fluid & 6 & $2(1)$ & 1 & 1 & 1 & 6 & 3 & 3 & 1 & 0 & 0 \\
\hline Ternary fluid & 7 & $2(1)$ & 1 & 2 & 3 & 10 & 6 & 6 & 3 & 0 & 0 \\
\hline Nematic & 7 & $5(3)$ & 2 & 0 & 1 & 3 & 1 & 1 & 0 & 3 & 1 \\
\hline $\begin{array}{l}\text { Smetic } A \\
\quad \text { and cholesteric }\end{array}$ & 6 & $5(3)$ & 2 & 1 & 1 & 6 & 3 & 3 & 1 & 1 & 0 \\
\hline $\begin{array}{l}\text { Smectic } B \\
\quad \text { (uniaxial) }\end{array}$ & 8 & $5(3)$ & 2 & 2 & 2 & 7 & 4 & 4 & 2 & 2 & 0 \\
\hline Uniaxial crystal & 8 & $5(3)$ & 2 & 2 & 2 & 8 & 5 & 5 & 3 & 0 & 0 \\
\hline Smectic $C$ & 7 & $13(9)$ & 4 & 2 & 2 & 6 & 3 & 3 & 1 & 9 & 2 \\
\hline General crystal & 8 & $21(15)$ & 6 & 9 & 6 & 28 & 21 & 21 & 15 & 0 & 0 \\
\hline Cubic crystal & 8 & $3(2)$ & 1 & 1 & 1 & 5 & 3 & 3 & 2 & 0 & 0 \\
\hline Glass & 8 & $2(1)$ & 1 & 1 & 1 & 4 & 2 & 2 & 1 & 0 & 0 \\
\hline
\end{tabular}

picture which does not distinguish between the two can be obtained by setting $\left\{\xi_{i}^{\alpha}\right\},\left\{\zeta^{\alpha \beta}\right\}$ equal zero.

To illustrate Table I observe that the incompressible isothermal simple fluid is characterized completely by only one viscous coefficient (the columns $\kappa_{i j}$ and $\xi_{i}{ }^{\alpha}$ have no meaning for isothermal systems), while the incompressible isothermal nematic phase has only three viscous coefficients, one dissipative parameter $\zeta^{\alpha \beta} \sim 1 / \gamma_{1}$, three Frank constants, and one flow parameter. Although it is harder to justify incompressibility and isothermal approximations for a smectic $A$ phase, a theory with these approximations would have three viscosities, one dissipative parameter, one first-order elastic constant, and one of second order. The increase in the number of first-order elastic constants from the uniaxial nematic phase, through the uniaxial smectic phases, to the uniaxial single crystal phase demonstrates quite clearly how one progresses through these phases by introducing various elastic restoring forces.

Smectic $\boldsymbol{C}$ liquid crystals which are biaxial have more dissipative parameters than the other smectic liquid crystals listed in Table I, and also more second-order elastic constants. However, their first-order elastic properties are exactly the same as those of a smectic $A$ liquid crystal. The cubic crystal has, in general, three isothermal elastic constants, one thermal-expansion parameter, and one independent heat capacity for the total of five shown in column (a). The compressible isothermal cubic crystal, on the other hand, has only three elastic constants.

The row listing the parameters for a glass is more phenomenological than the others. In a glass we believe it is possible to argue that the relaxation time for a local shear is astronomically long even though the glass is amorphous. Thus we may view a glass, for times short compared to the shear relaxation time but long compared to all other microscopic times, as possessing an additional "thermodynamic" variable, corresponding to isotropic volume-preserving stresses. If we allow for this extra "conserved variable" we must allow for the "thermodynamic derivative," which gives the shear elastic constant, and also for the two additional dissipative parameters $\xi$ and $\zeta$. As in a solid, we may look upon these modes as describing the diffusion of "vacancies" (e.g., dangling bonds in the accepted three-dimensional models of amorphous Si or Ge).

One of the simplest applications of Eqs. (6.1) is to solve an $N \times N$ secular determinant to obtain $N$ roots for $\omega(\vec{q})$. The qualitative nature of these $N$ solutions is rather easily inferred from the basic properties of the various systems. In fact, it is far easier to specify the number and type of modes that exist for a system than it is to count the number of parameters required to fully describe the system (Table I). Table II summarizes the quali- 
tative results for the number of extra variables and type of modes that occur in the various phases described in this paper. The square brackets about certain entries denote that they apply only in special directions of $\vec{q}$, where certain first-order elastic effects vanish and propagating modes may become diffusive. We say "may" because diffusive behavior is not required. The elastic constants and viscosities could lead to "underdamped" propagating modes with a dispersion law $\omega \sim q^{2}$ (as they also could, in principle, in a nematic phase). The necessary inequality for such propagating modes, spin waves, occurs in an isotropic ferromagnet but it is not anticipated in liquid crystals.

Note also that the propagating modes with $\omega=c q$ and $\omega=-c q$ are counted separately. Thus, the sum of the last two columns equals the $N$ that appears in Table I. Note finally that all "extra variables" in this table are even under time reversal. For a superfluid, by contrast, the superfluid phase is an extra variable that is odd under time reversal, and there are six modes, four propagating and two diffusive.

A more general application of Eqs. (6.1) is to extract the long-time, or low-frequency, behavior of the spectral densities,

$$
S_{\alpha \beta}(\omega) \equiv \int e^{-i \omega\left(t-t^{\circ}\right)}\left\langle\delta U_{\alpha}(t) \delta U_{\beta}\left(t^{\prime}\right)\right\rangle d\left(t-t^{\prime}\right),
$$

where the quantities $\left\{\delta U_{\alpha}(t)\right\}, \alpha=1,2, \ldots, N$ are the $N$ time-dependent hydrodynamic variables: $\rho$, $g_{i}, \epsilon,\left\{x^{\alpha}\right\}$. More specifically, we may use Eqs. (6.1) to calculate for each spatial Fourier component with wave vector $\overrightarrow{\mathrm{q}}$, the quantity $S_{\alpha \beta}(\overrightarrow{\mathrm{q}}, \omega)$. The procedure involves the fluctuation-dissipation theorem and the general theory of linear response functions. Details of these general theories are available elsewhere $\mathrm{e}^{1,12}$ and we only sketch their application to the present problem.

Assume that when $t \leq 0$, external forces $f_{\gamma}(\vec{q})$ are applied to disturb the system from homogeneous

TABLE II. Summary of the number and type of modes (Ref. 34). See the text for further explanation.

\begin{tabular}{|c|c|c|c|}
\hline Phase & $\begin{array}{c}\text { Extra variable (s) } \\
\text { (number) }\end{array}$ & $\begin{array}{l}\text { No. propagating } \\
\text { modes }\end{array}$ & $\begin{array}{l}\text { No. diffusive } \\
\text { modes }\end{array}$ \\
\hline Simple fluid & (0) & 2 & 3 \\
\hline Binary fluid & Concentration (1) & 2 & 4 \\
\hline Ternary fluid & Concentration (2) & 2 & 5 \\
\hline Nematic & Direction (2) & 2 & 5 \\
\hline $\begin{array}{l}\text { Smectic } A \\
\quad \text { and cholesteric }\end{array}$ & Displacement (1) & $4,[2]$ & $2,[4]$ \\
\hline Smectic $B$ & Displacement (3) & 6 , [4] & $2,[4]$ \\
\hline Smectic o & $\begin{array}{l}\text { Displacement (1) } \\
\quad \text { and direction }(1)\end{array}$ & $4,[2]$ & $3,[5]$ \\
\hline $\begin{array}{l}\text { Crystal } \\
\text { and glass }\end{array}$ & Displacement (3) & 6 & 2 \\
\hline
\end{tabular}

thermal equilibrium. The external forces appropriate to Eqs. (6.1) are, for example, $\delta T(\overrightarrow{\mathrm{q}})$, $\delta v_{j}(\overrightarrow{\mathrm{q}}), \delta \mu(\overrightarrow{\mathrm{q}})$, and $\delta \phi_{i}{ }^{\alpha}(\overrightarrow{\mathrm{q}})$. Then the initial deviations in the hydrodynamical variables from equilibrium are given by the linear relations

$$
\left.\delta U_{\alpha}(\overrightarrow{\mathrm{q}}, t)\right|_{t \leq 0}=\chi_{\alpha \gamma}(\overrightarrow{\mathrm{q}}, 0) f_{\gamma}(\overrightarrow{\mathrm{q}}),
$$

where

$$
\chi_{\alpha \gamma}(\overrightarrow{\mathrm{q}}, 0) \equiv\left(\frac{\partial U_{\alpha}(\overrightarrow{\mathrm{q}}, t)}{\partial f_{\gamma}(\overrightarrow{\mathrm{q}})}\right)_{t=0} .
$$

At time $t=0$ the external forces are turned off and the $\delta U_{\alpha}(\overrightarrow{\mathrm{q}}, t)$ for $t>0$ decay back to thermal-equilibrium values. This decay is described by Eqs.

(6.1) or (to simplify the discussion)

$$
\delta \dot{U}_{\alpha}(\overrightarrow{\mathrm{q}}, t)+\Lambda_{\alpha \beta}(\overrightarrow{\mathrm{q}}) \delta f_{\beta}(\overrightarrow{\mathrm{q}}, t)=0 .
$$

The Fourier transform of this equation, which is applicable only for $t>0$, yields

$$
-i \omega \delta U_{\alpha}(\overrightarrow{\mathrm{q}}, \omega)+\Lambda_{\alpha \beta}(\overrightarrow{\mathrm{q}}) \delta f_{\beta}(\overrightarrow{\mathrm{q}}, \omega)=\chi_{\alpha \beta}(\overrightarrow{\mathrm{q}}, 0) f_{\gamma}(\overrightarrow{\mathrm{q}}) .
$$

Defining the restoring forces $\delta f_{\beta}(\overrightarrow{\mathrm{q}}, \omega)$,

$$
\left[\delta U_{\alpha}(\overrightarrow{\mathrm{q}}, \omega) / \delta f_{\beta}(\overrightarrow{\mathrm{q}}, \omega)\right]=\chi_{\alpha \beta}(\overrightarrow{\mathrm{q}}, 0),
$$

in the same manner as for the initial external force [a justifiable step for the hydrodynamic problem in view of Eqs. (2.8), (2.15), etc.], we obtain

$$
\delta U_{\alpha}(\overrightarrow{\mathrm{q}}, \omega)=\left[-i \omega+\Lambda(\overrightarrow{\mathrm{q}}) \chi^{-1}(\overrightarrow{\mathrm{q}}, 0)\right]_{\alpha \beta}^{-1} \chi_{\beta \gamma}(\overrightarrow{\mathrm{q}}, 0) f_{\gamma}(\overrightarrow{\mathrm{q}}) .
$$

The general linear susceptibility $\chi_{\alpha \gamma}(q, \omega)$ is obtained from

$$
\left[\delta U_{\alpha}(\overrightarrow{\mathrm{q}}, \omega) / \delta f_{\gamma}(\overrightarrow{\mathrm{q}})\right]=(i / \omega)\left[\chi_{\alpha \gamma}(\overrightarrow{\mathrm{q}}, \omega)-\chi_{\alpha \gamma}(\overrightarrow{\mathrm{q}}, 0)\right],
$$

which gives

$$
\begin{aligned}
\chi_{\alpha \gamma}(\overrightarrow{\mathrm{q}}, \omega)-\chi_{\alpha \gamma}(\overrightarrow{\mathrm{q}}, 0) \\
\quad=i \omega\left[i \omega-\Lambda(\overrightarrow{\mathrm{q}}) \chi^{-1}(\overrightarrow{\mathrm{q}}, 0)\right]_{\alpha \beta}^{-1} \chi_{\beta \gamma}(\overrightarrow{\mathrm{q}}, 0) \\
\quad=\left[\chi^{-1}(\overrightarrow{\mathrm{q}}, 0)+\omega^{-1} \chi^{-1}(\overrightarrow{\mathrm{q}}, 0) i \Lambda(\overrightarrow{\mathrm{q}}) \chi^{-1}(\overrightarrow{\mathrm{q}}, 0)\right]_{\alpha \gamma}^{-1} .
\end{aligned}
$$

One important result may now be obtained with the aid of the fluctuation-dissipation theorem which states $^{35}$

$$
\begin{aligned}
S_{\alpha \beta}(\overrightarrow{\mathrm{q}}, \omega) & =2\left(k_{B} T / \omega\right) \chi_{\alpha \beta}{ }^{\prime \prime}(\overrightarrow{\mathrm{q}}, \omega) \\
= & -2\left(k_{B} T / \omega\right)(\text { diss pt }) \\
\times & {\left[\chi^{-1}(\overrightarrow{\mathrm{q}}, 0)+i \omega^{-1} \chi^{-1}(\overrightarrow{\mathrm{q}}, 0) \Lambda(\overrightarrow{\mathrm{q}}) \chi^{-1}(\overrightarrow{\mathrm{q}}, 0)\right]_{\alpha \beta}^{-1} . }
\end{aligned}
$$

Equations (6.10) and (6.11) show that the poles in the correlation functions correspond to the frequencies at which $\operatorname{det}\left[i \omega-\Lambda(\overrightarrow{\mathrm{q}}) \chi^{-1}(\overrightarrow{\mathrm{q}}, 0)\right]$ vanishes.

A second result is the identity (related to the Kramers-Kronig relation)

$$
\begin{aligned}
\chi_{\alpha \beta}(\overrightarrow{\mathrm{q}}, 0) & =\pi^{-1} \int d \omega \chi_{\alpha \beta}{ }^{\prime \prime}(\overrightarrow{\mathrm{q}}, \omega) / \omega \\
& =\left(2 \pi k_{B} T\right)^{-1} \int d \omega S_{\alpha \beta}(\overrightarrow{\mathrm{q}}, \omega) \\
& =\left(k_{B} T\right)^{-1}\left\langle\delta U_{\alpha}(\overrightarrow{\mathrm{q}}, t) \delta U_{\beta}(\overrightarrow{\mathrm{q}}, t)\right\rangle .
\end{aligned}
$$


For the system to be thermodynamically stable, the matrix $\left\langle\delta U_{\alpha}(\overrightarrow{\mathrm{q}}, t) \delta U_{\beta}(\overrightarrow{\mathrm{q}}, t)\right\rangle$ must be positive definite. From Eq. (6.7) this positive definite matrix is just the inverse of the positive definite matrix $\delta f_{B}(\vec{q}, \omega) / \delta U_{\alpha}(\vec{q}, \omega)$ that yields the stability criterion mentioned earlier. See, for example, Eq. (2.21) and the remarks following it.

A further general result follows from the hy drodynamic nature of these response functions. The matrix $\left[i \omega-\Lambda(\overrightarrow{\mathrm{q}}) \chi^{-1}(\overrightarrow{\mathrm{q}}, 0)\right]_{\alpha \beta}^{-1}$ can be written in a form that will be proportional to $\Pi_{\nu=1}^{N}[\omega$ $\left.-\omega_{\nu}(\vec{q})\right]^{-1}$, where the $\left\{\omega_{\nu}(\vec{q})\right\}$ are the hydrodynamic frequencies. From the fact that the $\left\{\omega_{\nu}\right\}$ vanish as $q \rightarrow 0$ one can justify, in the limit $q \rightarrow 0$ while $\omega$ remains finite, that the above hydrodynamic equations give

$$
\lim _{\vec{q} \rightarrow 0} S_{\alpha \beta}(\overrightarrow{\mathrm{q}}, \omega)=\left(2 k_{B} T / \omega^{2}\right) \lim _{\overrightarrow{\mathrm{q}} \rightarrow 0} \operatorname{Re}\left(\Lambda(\overrightarrow{\mathrm{q}})_{\alpha \beta}\right) .
$$

For those elements in $\Lambda(q)_{\alpha \beta}$ for which the limit does not vanish (i.e., in nematiclike systems the $\left\langle\delta n_{\alpha} \delta n_{\beta}\right\rangle$ fluctuations are of this type), one has

$\lim \lim _{\omega \rightarrow 0}\left(\omega^{2} / 2 k_{B} T\right) S_{\alpha \beta}(\overrightarrow{\mathrm{q}}, \omega)=\lim \lim _{\omega \rightarrow 0} \operatorname{Re} \Lambda(\overrightarrow{\mathrm{q}})_{\alpha \beta}$ $\lim _{\omega \rightarrow 0} \quad \vec{a} \rightarrow 0$ (6.14) (which for nematic liquid crystals is just $\gamma_{1}{ }^{-1}$ ). For the more usual hydrodynamic variables this limit vanishes as $q^{2}$, and we find

$\lim _{\omega \rightarrow 0} \lim _{\overrightarrow{\mathrm{q}} \rightarrow 0}\left(\omega^{2} / 2 k_{B} T q^{2}\right) S_{\alpha \beta}(\overrightarrow{\mathrm{q}}, \omega)=\lim _{\omega \rightarrow 0} \lim _{\overrightarrow{\mathrm{a}} \rightarrow 0} \operatorname{Re} \Lambda(\overrightarrow{\mathrm{q}})_{\alpha \beta} / q^{2}$.

Thus, for example, the $\left\langle v_{i} v_{j}\right\rangle$ correlations yield the viscosity coefficients $\eta_{i j k l}$. Although there are subtleties in the $q \rightarrow 0, \omega \rightarrow 0$ limits they do not enter into the tedious but straightforward determination of the measured quantities $S_{\alpha \beta}(\vec{q}, \omega)$.

Equations (6.14) and (6.15) are known as the Kubo formulas and they express rather general properties of many-body systems. We believe that it is preferable for a hydrodynamic theory of liquid crystals, like any other hydrodynamic theory, to manifest these properties. Specifically, as we spell out in more detail in Appendix B, we believe that it is unfortunate and deceptive to treat $\gamma_{2}$ in the hydrodynamics of nematic liquid crystals as a "dissipative parameter."

In addition to our own previous papers $\mathrm{s}^{9,11}$ on the subject and those of Lubensky, ${ }^{5}$ there have been several discussions with which we are in complete accord. In some of these, certain properties were intentionally omitted. For example, in the treatment of smectic liquid crystals by de Gennes, ${ }^{14}$ dissipation was neglected, and in various papers on nematic liquid crystals, assumptions about incompressibility were, with some justification, employed. ${ }^{20}$ It is of course possible by the latter restriction to limit the discussion to nonpropagating modes, modes whose frequency is of order $q^{2}$ and not of order $q$ when $q$ is small. One does so, however, only with the loss of one of the simplest and clearest properties of hydrodynamics, conservation of the number of modes.

We find it difficult to understand the justification for the oft-made approximation that retains modes with finite frequencies $\omega \sim-i / \tau$ and simultaneously discards as too high frequency a sound wave for which $\omega \sim c q$ can be arbitrarily slow. (Such a mode is always retained when one includes the "inertia" of the director, or equivalently when one writes a separate conservation law for the internal angular momentum.) Theories of liquid crystals that describe internal relaxation in this form are conjectural and although they may sometimes agree with experiment, they may very well not. Disagreement with experiment, for theories of this type, would not force any profound readjustment in our understanding of the material properties. Hydrodynamics in the sense we use the word here is less ambitious than many of the "conjectural" theories presented, but, on the other hand, when it disagrees with experiment one must face the fact that either the experiment was improperly performed or that the basic thermodynamic properties of the material have been misunderstood.

There have been several attempts to "generalize" earlier hydrodynamic theories of liquid crystals which we believe are not, in fact, generalizations, but incomplete and inconsistent extensions which have no basis and are incorrect in the hy drodynamic sense. ${ }^{17}$ This same criticism applies with equal force to the discussions of chemical processes or internal relaxation in standard references on irreversible thermodynamics. ${ }^{36}$ Phenomenological irreversible theories which make hypotheses of this type may well be consistent with their hypotheses, but in contrast with hydrodynamic theories there is no reason why they must apply in the real world. Their predictions may be qualitatively and are likely to be quantitatively in contradiction with experiment.

\section{ACKNOWLEDGMENTS}

A number of the ideas and calculations contained herein were developed in connection with nematic liquid crystals in studies performed jointly with J. Swift, T. Lubensky, and D. Forster. We are especially indebted to P. G. de Gennes for repeated discussions that contributed to our current formulation of these problems. Conversations with B. Halperin and A. Rapini are also gratefully acknowledged.

\section{APPENDIX A: GENERAL ARGUMENTS CONCERNING SYMMETRIC STRESS TENSORS}

In this appendix we recall the argument ${ }^{37}$ which states that if the total angular momentum is con- 
served, the momentum density $g_{i}{ }^{0}$ satisfies $\dot{g}_{i}{ }^{0}$ $+\partial_{j} \sigma_{i j}{ }^{0}=0$, and $\sigma_{i j}{ }^{0}$ is not symmetric, then it is always possible to choose a new $g_{i}$ and a new $\sigma_{i j}$ such that
(i) $\dot{\rho}+\partial_{j} g_{j}=0$,
(ii) $\int d \overrightarrow{\mathrm{r}} g_{i}=\int d \overrightarrow{\mathrm{r}} g_{j}{ }^{0}$,
(iii) $\dot{g}_{i}+\partial_{j} \sigma_{i j}=0$,
(iv) $\sigma_{i j}=\sigma_{j i}$,

and the conservation of angular momentum is expressed by

$$
\text { (v) }\left(r_{i} \dot{g}_{j}-r_{j} \dot{g}_{i}\right)+\partial_{k}\left(r_{i} \sigma_{j k}-r_{j} \sigma_{i k}\right)=0 \text {. }
$$

All physical properties may be calculated with equal ease using the new stress tensor in terms of which angular momentum conservation is a tautology and thus introduces no new modes, parameters, or dynamical properties.

The proof is an exercise in arithmetic. We begin with separate statements for the conservation of angular momentum,

$$
\frac{\partial}{\partial t}\left(r_{i} g_{j}{ }^{0}-r_{j} g_{i}{ }^{0}+\epsilon_{i j k} s_{k}\right)=\partial_{k} M_{i j k}{ }^{0},
$$

where $s_{k}$ represents some internal angular momentum of the particles, and for the conservation of linear momentum,

Since

$$
\dot{g}_{i}{ }^{0}=-\partial_{j} \sigma_{i j}{ }^{0} \text {. }
$$

$-r_{i} \partial_{k} \sigma_{j k}{ }^{0}+r_{j} \partial_{k} \sigma_{i k}{ }^{0}$

$$
=\partial_{k}\left(-r_{i}{\sigma_{j k}}^{0}+r_{j} \sigma_{i k}{ }^{0}\right)+\sigma_{j i}{ }^{0}-\sigma_{i j}{ }^{0},
$$

the antisymmetric part of $\sigma_{j i}{ }^{0}+\frac{1}{2} \epsilon_{i j k} s_{k}$, i.e.,

$$
\begin{aligned}
\sigma_{j i}{ }^{0}-\sigma_{i j}{ }^{0}+\epsilon_{i j k} \dot{s}_{k} & =\partial_{k}\left(M_{i j k}{ }^{0}+r_{i} \sigma_{j k}{ }^{0}-\gamma_{j} \sigma_{i k}{ }^{0}\right) \\
& \equiv-2 \partial_{k} f_{i j k},
\end{aligned}
$$

is a divergence. By construction, the quantity

$$
\sigma_{i j} \equiv \sigma_{i j}{ }^{0}-\frac{1}{2} \epsilon_{i j k} \dot{s}_{k}-\partial_{k} f_{i j k}+\partial_{k} f_{i k j}+\partial_{k} f_{j k i}
$$

is symmetric. Furthermore,

$$
g_{i} \equiv g_{i}{ }^{0}+\frac{1}{2} \partial_{j} \epsilon_{i j k} s_{k}
$$

has the properties that $\int g_{i}=\int g_{i}{ }^{0}$ and $\partial_{i} g_{i}=\partial_{i} g_{i}{ }^{0}$ $=-\dot{\rho}$. Substituting, we obtain

$$
\begin{aligned}
\dot{g}_{i} & =\dot{g}_{i}{ }^{0}+\frac{1}{2} \partial_{j} \epsilon_{i j k} \dot{s}_{k} \\
& =-\partial_{j} \sigma_{i j}-\partial_{j} \partial_{k} f_{i j k}+\partial_{j} \partial_{k} f_{i k j}+\partial_{j} \partial_{k} f_{j k i} .
\end{aligned}
$$

The second and third terms on the right-hand side cancel and the last term vanishes because $f_{j k i}$ is antisymmetric in $j$ and $k$. We therefore have

$$
\text { and } \begin{aligned}
& \dot{g}_{i}=-\partial_{j} \sigma_{i j} \\
& \quad\left(r_{i} \dot{g}_{j}-r_{j} \dot{g}_{i}\right)=\partial_{k}\left(-r_{i} \sigma_{j k}+r_{j} \sigma_{i k}\right)
\end{aligned}
$$

$$
=\partial_{k}\left[M_{i j k}{ }^{0}+\frac{1}{2}\left(r_{i} \epsilon_{j k l} \dot{s}_{l}-r_{j} \epsilon_{i k l} \dot{s}_{l}\right)\right] .
$$

The significance of these manipulations is the following. It is impossible to really distinguish between intrinsic angular momentum and the orbital angular momentum of the particles that make up a molecule. ${ }^{38}$ Alternatively, it is always possible subtract the derivative of any intrinsic angular momentum from the linear momentum density without changing either its volume integral or its divergence. At a microscopic level this can have no effect. The difference between the stress tensors and the momentum densities is a microscopic quantity whose (microscopic) relaxation cannot be predicted rigorously. The different stress tensors also have different values on boundaries, but, in fact, those have no effect and do not even occur in a calculation since both of them give the same value to $\partial_{j} \sigma_{i j}=-\dot{g}_{i}=0$ in equilibrium.

We can get another insight into the difference by noting that in the microscopic theory we may write

$$
\begin{aligned}
g_{i}{ }^{\mathrm{mol}}= & \sum_{\text {atom }} p_{i}{ }^{\text {atom }} \delta\left[\overrightarrow{\mathrm{r}}-\overrightarrow{\mathrm{r}}^{\text {atom }}(t)\right] \\
= & \sum_{\text {atom }} p_{i}{ }^{\text {atom }}\left\{\delta\left[\overrightarrow{\mathrm{r}}-\overrightarrow{\mathrm{r}}^{\mathrm{mol}}(t)\right]\right. \\
& \left.-\left[\overrightarrow{\mathrm{r}}^{\text {atom }}(t)-\overrightarrow{\mathrm{r}}^{\mathrm{mol}}(t)\right]_{j} \nabla_{j} \delta\left[\overrightarrow{\mathrm{r}}-\overrightarrow{\mathrm{r}}^{\mathrm{mol}}(t)\right]+\cdots\right\} \\
= & g_{i}{ }^{0 \text { mol }}+\frac{1}{2} \sum_{\text {atom }}\left\{\nabla \times\left[\left(\overrightarrow{\mathrm{r}}^{\text {atom }}-\overrightarrow{\mathrm{r}}^{\mathrm{mol}}\right) \times \overrightarrow{\mathrm{p}}^{\text {at om }}\right]\right. \\
& \left.\times \delta\left[\overrightarrow{\mathrm{r}}-\overrightarrow{\mathrm{r}}^{\mathrm{mol}}(t)\right]\right\}_{i}+\nabla\{\cdots\},
\end{aligned}
$$

in which case the second term plays the role of the internal angular momentum. ${ }^{39}$ There appears to be no reason for prefering $g_{i}{ }^{0 \mathrm{~mol}}$ to $g_{i}{ }^{\mathrm{mol}}$ on a macroscopic level and all the physical consequences follow just as simply when we use

$$
\sum_{\mathrm{mol}} g_{i}{ }^{\mathrm{mol}}=g_{i} .
$$

Indeed, the symmetric stress tensor comes closer to describing the microscopic situation and the large forces that must be supplied to keep large quantities of liquid crystals in a distorted position.

Finally we remark that the absence of a mode related to the angular momentum density is connected with its nonlocal character - the reference to an unbounded and irrelevant moment arm when the system is unbounded in extent.

\section{APPENDIX B: REMARKS ON PHYSICAL SIGNIFICANCE OF PARAMETERS $\gamma_{1}$ AND $\gamma_{2}$}

We have argued that the writing of the director equation as a frictional equation, labeling $\gamma_{2}$ as a dissipative coefficient, is not the most desirable or natural procedure from a microscopic or even a macroscopic point of view. We would like to explain the reasoning behind this argument by using the same artificial parametrization in an analo- 
gous, more familiar example from ordinary fluids. For slowly varying disturbances it is always possible to write

$$
\delta \dot{s}=\left(\frac{\partial s}{\partial T}\right)_{\rho} \delta \dot{T}+\left(\frac{\partial s}{\partial \rho}\right)_{T} \delta \dot{\rho}
$$

and, furthermore, using $T d s+\left(p / \rho^{2}\right) d \rho=d(\epsilon / \rho)$ and $\rho=-\nabla_{i} \rho v_{i}$,

$$
\delta \dot{s}-\left(\frac{\partial p}{\partial T}\right)_{\rho} \rho^{-2} \nabla_{i} \rho v_{i}=\frac{C_{v}}{T} \delta \dot{T}
$$

If we use the known correct result for the heatdiffusion modes, i.e., $\dot{T}=\left(\kappa / \rho C_{p}\right) \nabla^{2} T$, we may write

or

$$
\kappa^{-1} T \rho \dot{\delta} \dot{s}-\left(\frac{\partial p}{\partial T}\right)_{\rho} \kappa^{-1} T \nabla_{i} \delta v_{i}=\left(\frac{C_{v}}{C_{p}}\right) \nabla^{2} \delta T
$$

$$
\bar{\gamma}_{1} \delta \dot{s}-\bar{\gamma}_{2} \nabla_{i} v_{i}=\left(C_{v} / C_{p}\right) \nabla^{2} \delta T .
$$

In fact, this parametrization is essentially identical to the one that has been introduced for the director; i.e., $\bar{\gamma}_{1}^{-1}$ is essentially the transport coefficient and $\bar{\gamma}_{2}=\bar{\gamma}_{1} \rho^{-1}(\partial p / \partial T)_{\rho}$. The fact that $\bar{\gamma}_{2}$ is not a transport coefficient but that $\bar{\gamma}_{2} / \bar{\gamma}_{1}$ is a thermodynamic derivative, $\rho^{-1}(\partial p / \partial T)$, has many consequences.

(i) $\bar{\gamma}_{2} \rightarrow \infty$ and $\bar{\gamma}_{1}{ }^{-1} \rightarrow 0$ such that $\rho^{-1}(\partial p / \partial T)$ is finite is a possible limit. Its value $\left(\bar{\gamma}_{2} / \bar{\gamma}_{1}\right)$ can be measured in a static experiment in which there is no entropy production.

(ii) $\bar{\gamma}_{2}$ always appears as a square in the expression for the normal modes, i. e.,

$$
\begin{aligned}
c^{2} & =\left(\frac{\partial p}{\partial \rho}\right)_{s}=\left(\frac{\partial p}{\partial \rho}\right)_{T}+\frac{1}{\rho^{2}}\left(\frac{\partial p}{\partial T}\right)_{\rho}^{2}\left(\frac{\partial T}{\partial s}\right)_{\rho} \\
& =\left(\frac{\partial p}{\partial \rho}\right)_{T}+\left(\frac{\bar{\gamma}_{2}}{\bar{\gamma}_{1}}\right)^{2}\left(\frac{\partial T}{\partial s}\right)_{\rho},
\end{aligned}
$$

and its sign cannot be determined in this fashion.

(iii) The sign of $\bar{\gamma}_{2} / \bar{\gamma}_{1}$ and its magnitude are arbitrary (i.e., not determined by any general principle) and $\bar{\gamma}_{2}$ does not appear in the entropy production.

The claim that $\bar{\gamma}_{1}^{-1}$ is the proper Onsager coefficient for the dissipation is also in accord with the expectation that were it not for the coupling to compression, the frequency of this temperature mode would be $\bar{\gamma}_{1}^{-1}\left(C_{v} / T\right)^{-1}$, where $\bar{\gamma}_{1}^{-1}$ is the Onsager coefficient and

$$
\left(\frac{\partial S}{\partial T}\right)_{\rho}=\left(\frac{C_{v}}{T}\right)=-\left(\frac{\partial^{2} F}{\partial T^{2}}\right)
$$

the second derivative of the free energy per unit mass, i.e., the susceptibility. The connection between the parameters $\bar{\gamma}_{1}{ }^{-1}$ and $\gamma_{1}{ }^{-1}$ and between $\left(C_{v} / T\right)$ and the inverse Frank constant $K^{-1}$, like that between $(d p / d T)_{\rho}$ and $\left(-\gamma_{2}+1\right) / \gamma_{1}$, is apparent. The difference manifests itself in various microscopic fashions. For example, there is no Kubo formula (in term of currents) for $\gamma_{2}$ or $\gamma_{1}$ although there is one for $\gamma_{1}^{-1}$ :

$$
\begin{aligned}
\gamma_{1}{ }^{-1} & \left.=\lim _{\omega \rightarrow 0} \lim _{\vec{a} \rightarrow 0} \chi_{\dot{n}, \dot{n}}^{\prime \prime} \cdot \vec{q}, \omega\right) / \omega \\
& =\lim _{\omega \rightarrow 0} \lim _{\vec{q} \rightarrow 0}\left(\omega^{2} / 2 k_{B} T\right) S_{n, n}(\vec{q}, \omega) .
\end{aligned}
$$

There is also a thermodynamic relation for the analog of the thermal expansion,

$$
\lim _{\vec{q} \rightarrow 0} \lim _{\omega \rightarrow 0} \chi_{n_{1}} \sigma_{13}(\vec{q}, \omega),
$$

from which one determines $\gamma_{2} / \gamma_{1}=-\lambda$. There is one subtle detail concerning the correlation function in Eq. (B7) that is discussed separately in Appendix C.

In the same way as there is a phenomenological qualitative relation between translational selfdiffusion, a nonhydrodynamic process, and viscosity (Stokes's law) which states

$$
D \approx k_{B} T / 6 \pi \eta^{a},
$$

there is a phenomenological expression that relates a "rotational-diffusion" model for the relaxation of molecular orientation and the viscosity \{i.e., $\left[\partial / \partial t-D_{\text {rot }}\left(\partial^{2} / \partial \theta^{2}\right)\right]$ (orientation $\left.)=0\right\}$, namely,

$$
D_{\text {rot }} \approx k_{B} T / \eta a^{3},
$$

where $a$ is a typical "molecular dimension."

The usual parametrization of the director mode is very much in this spirit. The smallness of the diffusion constant for the director brings to mind the smallness of the mutual diffusion constant near the critical point of binary mixture. Near this point, the effect of coupled modes is extremely important and gives rise to a very long effective relaxation time. It seems likely that the same mechanism may be responsible for the anomalous sound attenuation at low frequencies in nematic liquid crystals. ${ }^{40}$

\section{APPENDIX C: DETAILED DISCUSSION OF RELATION BETWEEN $\mu_{i j k}$ AND CORRELATION FUNCTIONS}

There is a subtlety involved in infinite systems that might be of interest to some readers. Imagine, for example, in a nematic phase that in place of equations like (2.17) the reversible part of $\delta n_{i}(\overrightarrow{\mathrm{q}})$ were characterized by where

$$
\delta \dot{n}_{i}(\overrightarrow{\mathrm{q}})=A_{i j}(\overrightarrow{\mathrm{q}}) \delta v_{j}(\overrightarrow{\mathrm{q}})
$$

$$
A_{i j}(\overrightarrow{\mathrm{q}}) \equiv \chi_{\dot{n}_{i}, g_{j}}(\overrightarrow{\mathrm{q}}, 0)=\pi^{-1} \int d \omega \chi_{\dot{n}_{i}, q_{j}}^{\prime \prime}(\overrightarrow{\mathrm{q}}, \omega) / \omega .
$$

Employing, successively, the invariance in time

$$
\chi_{\dot{n}_{i, g_{j}}}^{\prime \prime}(\overrightarrow{\mathrm{q}}, \omega)=\chi_{n_{i}, \dot{\dot{B}_{j}}}^{\prime \prime}(\overrightarrow{\mathrm{q}}, \omega) \text {, }
$$

momentum conservation

$$
\chi_{n_{i}, \dot{\xi}_{j}}^{\prime \prime}(\vec{q}, \omega)=i \chi_{n_{i}, \sigma_{j k}}^{\prime \prime}(\vec{q}, \omega) q_{k},
$$


and the fluctuation-dissipation theorem [Eq. (6.11)], we obtain

$$
A_{i j}(q)=\left(2 \pi k_{B} T\right)^{-1} \int d \omega S_{n_{i}, \sigma_{j k}}(\overrightarrow{\mathrm{q}}, \omega) i q_{k} .
$$

The quantity $\mu_{i j k}$, introduced in Eq. (2.17), is thus formally defined as

$$
\mu_{i j k}=\left(2 \pi k_{B} T\right)^{-1} \int d \omega S_{n_{i}, \sigma_{j k}}(\overrightarrow{\mathrm{q}} \omega) .
$$

If $n_{i}$ and $\sigma_{j k}$ had only short-range correlations, that is, if $\mu_{i j k}$ were truly a local quantity, there would be only one constant,

$$
\mu_{i j k}=\frac{1}{2}(\lambda+1)\left(\delta_{i j} n_{k}^{0}+\delta_{i k} n_{j}^{0}\right), \quad i \neq j
$$

that must be symmetric in $j k$. This would imply a relation

$$
\delta \dot{n}_{i}=\frac{1}{2}(1+\lambda)\left(\nabla_{i} v_{3}+\nabla_{3} v_{i}\right) \text { for } i=2 \text { and } 3
$$

in place of Eq. (2.17). The fact that $n_{i}$ and $\sigma_{j k}$ have long-range correlations can be shown to follow from the known effects of rotating the system. Specifically the effect of a uniform rotation is

$$
\begin{aligned}
\epsilon_{k j l}\left(\frac{\partial}{\partial q_{k}}\right) A_{i j}(q) & =\pi^{-1} \int d \omega \chi_{n_{i},\left(r_{k} \varepsilon_{j} \epsilon_{l j k}\right)}^{\prime \prime}(\overrightarrow{\mathrm{q}}, \omega) \\
& =\epsilon_{l i p} n_{p}^{0} .
\end{aligned}
$$

Thus there is an additional term in Eq. (C7) of the form

$$
-\left[\left(q_{i} q_{j} n_{k}^{0}+q_{i} q_{k} n_{j}^{0}\right)-\left(n_{l}^{0} q_{l}\right) q_{i} \delta_{j k}\right] q^{-2}
$$

which implies Eq. (2.17), i.e.,

$$
\delta \dot{n}_{i}=-\frac{1}{2}\left[n^{0} \times(\nabla \times v)\right]_{i}+\frac{1}{2} \lambda\left(\nabla_{i} v_{3}+\nabla_{3} v_{i}\right)
$$

for $i=1$ or 2 .

The fact that this extra term exists implies, as mentioned above, that the stress tensor and the director have infinite range correlations. This should not be surprising since, for example, application of a uniform magnetic field, at an arbitrary angle to the director, produces a stress in the fluid which (assuming dislocations do not appear) will have to be balanced by the forces (or torques) exerted at the boundary.

The situation is quite parallel to one that arises in a superfluid. There it is the current-current correlations that have an infinite range, and their correlation functions depend on direction as $q \rightarrow 0$. Likewise, there is a tendency to produce vortices at vanishing small rotational rates in large samples.
*Work supported in part by the National Science Foundation under Grant No. GP 16504, by the Advanced Research Projects Agency under contract No. DAHC-1567-C-0219, and by the Joint Services Electronics Program under Contract No. N0014-67-A-0298-0006.

†Guggenheim Fellow 1971-1972.

†Laboratoire associé au C.N.R.S.

$\S$ National Science Foundation Senior Postdoctoral Fellow 1971-1972.

${ }^{1}$ L. P. Kadanoff and P. C. Martin, Ann. Phys. (N.Y.) 24, 419 (1963).

${ }^{2}$ B. I. Halperin and P. C. Hohenberg, Phys. Rev. $\underline{188}$ 898 (1969).

${ }^{3}$ The first suggestion of this possibility was made by G. S. Rushbrooke and P. J. Wood, Mol. Phys. 1, 257 (1958); recently the problem has been studied by V. L. Berezhinski, Zh. Eksperim. i Teor. Fiz. 59, 907 (1970) [Sov. Phys. JETP 32, 493 (1971)].

${ }^{4}$ The absence of order of the type present in these systems is commented upon by L. Landau and E. M. Lifschitz, Statistical Physics (Addison-Wesley, Reading, Mass., 1959), p. 411.

${ }^{5}$ T. Lubensky, Phys. Rev. A $\underline{6}, 452$ (1972); C. Fan, L. Kramer, and M. Stephen, ibid. 2, 2482 (1970), have also discussed cholesterics.

${ }^{6}$ (a) L. Landau and' E. M. Lifschitz, Fluid Mechanics (Addison-Wesley, Reading, Mass., 1959), p. 219; (b) P. C. Martin, in Statistical Mechanics of Equilibrium and NonEquilibrium, edited by J. Meixner (North-Holland, Amsterdam, 1965), pp. 122-124.

${ }^{7}$ Reference 6 (a), p. 507; P. C. Hohenberg and P. C. Martin, Ann. Phys. (N. Y.) 34, 291 (1965).

${ }^{8} \mathrm{~K}$. H. Michel and F. Schwabl, Physik Kondensierten Materie 11, 144 (1970).

${ }^{9}$ D. Forster, T. C. Lubensky, P. C. Martin, J. Swift, and P. S. Pershan, Phys. Rev. Letters 26, 1016 (1971). Hereafter referred to as FLMPS.

${ }^{10} \mathrm{C}$. Herring, J. Appl. Phys. 21, 437 (1950).

${ }^{11}$ P. C. Martin, P. S. Pershan, and J. Swift, Phys. Rev.Letters 25,844 (1970). Hereafter referred to as MPS. In addition to this incomplete theory it seems appropriate to comment here on the related work of $\mathrm{H}$. Schmidt and J. Jähnig [Ann. Phys. (N.Y.) 71, 129 (1972)] (SJ) which we believe suffers from similar but less severe difficulties. SJ contains a proper framework for the reactive properties of crystals and liquid crystals, as does MPS. In addition it contains many dissipative coefficients not contained in MPS, With these coefficients, $\mathrm{SJ}$, in contrast to MPS, can properly describe a compressible nematic liquid crystal. If they did not, for convenience, drop thermal effects, their results would have the same content as FLMPS. However, like MPS, it seems to us that SJ do not take sufficient account of the connection between continuous broken symmetries and hydrodynamic parameters. As a result, SJ miss hydrodynamic parameters that describe vacancy diffusion, and which appear, from our point of view, in the same fashion and for the same reason as the parameter omitted in MPS for nematics. SJ also contains irrelevant phenomenological parameters which describe, perhaps inaccurately, modes that relax quickly. The two objections are independent. The formalism of SJ could certainly be extended to include vacancy diffusion although the need to do so is far less apparent without making a connection with broken symmetries. Once this has been done it is certainly possible to eliminate the implicit and possibly incorrect nonhydrodynamic predictions. (SJ have done so for nematic liquid crystals.) Presumably, if similar tedious operations were carried out with the extended equations, hydrodynamic equations equivalent to ours would finally 
emerge. A third, less important difference concerns form not substance. By taking parameters which reflect microscopic symmetries under time reversal, etc., we are led to equations which are considerably more concise, and which manifest stability (positivity) and symmetry properties more clearly. This difference between FLMPS and SJ (and to a greater extent, the earlier continuummechanics approaches) is best seen by examining the final expressions for the modes in nematics. These differences would probably be more accentuated for the more complex systems whose equations are obtained here.

${ }^{12}$ P. C. Martin, in Many Body Physics, edited by C. De Witt and R. Balian (Gordon and Breach, New York, 1968).

${ }^{13}$ The transformation of the quantities in Eq. (2.1) from the laboratory frame to the rest frame will not be considered here. Note, however, that there is a difference: $\left(\sigma_{i j}\right)_{1 a b}-\left(\sigma_{i j}\right)_{\text {rest }}=\rho v_{i} v_{j}$, and others often reserve the symbol $\sigma_{i j}$ for $\left(\sigma_{i j}\right)_{\text {rest }}$.

${ }^{14} \mathrm{P}$. G. de Gennes, J. Phys. Suppl. 30, 65 (1969). For arbitrary directions of propagation, and neglecting dissipative effects, the probable existence of two pairs of propagating modes in smectic $A$ phase was first pointed out by de Gennes.

${ }^{15}$ For crystals consisting of more than a single atomic (or molecular) species there are separate conservation laws for the mass density of each of the species. Thus a diatomic crystal has a total of nine hydrodynamic modes, a triatomic has ten, etc.

${ }^{16} \mathrm{P}$. G. de Gennes and G. Sarma, Phys. Letters $\underline{38 \mathrm{~A}}$, 219 (1972).

${ }^{17}$ In the hydrodynamic regime for nematics, the "extension" of H. W. Hwang, Phys. Rev. Letters 26, 1525 (1971), is equivalent to FLMPS. Outside of the hydrodynamic regime, the terms he keeps in addition are $a d$ hoc and incomplete and there is no reason to think experiments would necessarily give the line shapes they predict even if the experiments could be performed. They are just the "irrelevant transport coefficients" which should be discarded as discussed in Ref. 11. Some readers may object to our use of the word irrelevant, since under certain circumstances nonhydrodynamic modes are slow and measurable, e.g., near phase transitions. We agree but point out in response that the same arguments apply in such cases to other variables that have been omitted (e.g., to the magnitude of the order parameter as well as its direction).

${ }^{18}$ Note that for hydrodynamic variations one proves the positivity of $R$ from thermodynamics and dynamics. It is not an additional postulate; see, for example, Refs. 1 and 12 .

${ }^{19}$ Orsay Liquid Crystal Group, J. Chem. Phys. $\underline{51}, 816$ (1969); Phys. Rev. Letters 22, 1361 (1969); O. Parodi, J. Phys. 31, 581 (1970).

${ }^{20}$ J. L. Ericksen, Arch. Ratl. Mech. Anal. 4, 231 (1960); Phys. Fluids 9, 1205 (1966); F. M. Leslie, Quart. J. Mech. Appl. Math. 19, 356 (1966); Arch. Ratl. Mech. Anal. 28, 265 (1968).

${ }^{21}$ F. C. Frank, Discussions Faraday Soc. $\underline{25}, 19$ (1958).

${ }^{22}$ The constitutive equations for nematic liquid crystals and the modes that follow are given in FLMPS. The translation between different notations is also spelled out in that letter.

${ }^{23}$ Except for the terms in the second row and column, this matrix is equivalent to the one involving $A_{0}, B_{0}, C_{0}$, and $K$ in Ref. 14. The terms in the second row and column are only important for the nonpropagating modes and the attenuation of the propagating ones. Thus when attenuation is neglected [as in (3.12) ] the result is identical to (III. 7) of Ref. 14. In this matrix the variables $\nabla_{i} x$ and $\nabla_{j} x$ for $i \neq j$ are treated as independent variables; i.e., $\left(\partial / \partial \nabla_{i} x\right)$ is taken at constant $\nabla_{j} x(i \neq j)$.

${ }^{24}$ The viscosities labeled $\nu_{2}$ to $\nu_{5}$ in FLMPS are here called $\eta_{2}$ to $\eta_{5}$. The viscosity $\eta_{1}=2\left(\nu_{1}+\nu_{5}\right)-\nu_{4}+\nu_{2}$ is chosen here in the more conventional fashion.

${ }^{25}$ The expression in Ref. 6, p. 124 is "simple" because it corresponds to the case where there are only four coupled longitudinal modes. In other directions, there is no separation into transverse and longitudinal.

${ }^{26}$ Phenomena of this type have previously been reported by O. Lehmann [Ann. Physik 2, $649(1900)]$ and discussed by C. W. Oseen [Trans. Faraday Soc. 29, 883 (1933)]; F. M. Leslie, Proc. Roy. Soc. (London) A307, 359 (1968).

${ }^{27}$ Orsay Liquid Crystals Group, Solid State Commun. 9, 653 (1971). Thermal properties are not included in this paper; thus the distinction between $(\partial p / \partial \rho)_{s}$ and $(\partial p / \partial \rho)_{T}$ is not made. See also H. Sackmann and D. Demus, Mol. Crystals $\underline{2}, 1$ (1966); T. R. Taylor, J. L. Fergason, and S. I. Arora, Phys. Rev. Letters 24, 359 (1960).

${ }^{28}$ The counting is like that for the elastic properties of a crystal of the same symmetry.

${ }^{29}$ These are the four B's in the first paper in Ref. 27. They were first discussed by A. Saupe, Mol. Cryst. Liquid Cryst. 7, 59 (1969).

${ }^{30}$ These are the three $A$ 's of Ref. 27.

${ }^{31}$ These are the two C's of Ref. 27.

${ }^{32}$ The first coefficient can be taken to be unity for the same reason previously given in the case of a nematic.

${ }^{33}$ There are, of course, additional second-order constants which are always masked by first-order terms.

${ }^{34} \mathrm{As}$ noted in Ref. 15, this count is only valid for monatomic, or monomolecular, liquid crystals, crystals, and glasses.

${ }^{35}$ H. Nyquist, Phys. Rev. $\underline{32}, 110$ (1928); H. B. Callen and T. R. Welton, ibid. 83,34 (1951), or see Refs. 1 and 12. The term "diss pt" gives the dissipative part. It 's the imaginary part when $\alpha$ and $\beta$ have the same timereversal properties and the real part when they do not.

${ }^{36} \mathrm{~S}$. de Groot and P. Mazur, Non-Equilibrium Thermodynamics (North-Holland, Amsterdam, 1962).

${ }^{37}$ This argument is reputed to be due to Belinfante in a more general relativistic context.

${ }^{38}$ If for some reason the coupling between "spin" and orbital angular momentums vanishes, or can be neglected, a separate conservation for "spin" angular momentum will follow from the microscopic Hamiltonian. This is actually the case for a number of models employed to describe magnetic problems.

${ }^{39}$ The argument is, of course, reminiscent of the one for the multipole expansion. It happens to suffer from irrelevant academic difficulties if there is dissociation, etc., since it requires that the molecules be treated as having fixed constituents. The main point is that nothing is gained by introducing the molecular centers of mass.

${ }^{40}$ M. E. Mullen, B. Lüthi, and M. J. Stephen, Phys. Rev. Letters 28, 799 (1972); A. L. Lord and M. M. Labes, ibid. $\underline{25}, 570$ (1970). 